\title{
Un historien sur le banc des accusés : Liu Zhiji juge Wei Shou
}

\section{Damien Chaussende *}

\author{
Abstract
}

\begin{abstract}
An historian in the dock: Liu Zhiji on Wei Shou
In Liu Zhiji’s Generalities on History (Shitong 史通), Wei Shou 魏收 (506-572) appears to be the counter-model of the ideal historian. Judged as partial, unfair and servile to the political power that employed him (the Northern Qi), Wei Shou, author of the Book of Wei (Weishu 魏書), is indeed the bugbear of the critic. The article examines the formal and ideological particularities of the Book of Wei, as brought out by Liu Zhiji 劉知幾 (661-721). The numerous comments of Liu Zhiji shows that the question of political legitimity, especially the antagonism Northern states vs Southern states, count for much in his judgements on the historical works of and on the Early Medieval period (220-589).
\end{abstract}

\section{首三德 \\ 被告席上的史學家 : 劉知幾筆下的魏收}

在《史通》裡, 魏收 (506-572) 是理想模範史學家的反面榜樣。《史通》的 作者劉知幾 (661-721) 認為魏收在他所著的《魏書》裡對他的雇主北齊政權 顯得卑躬屈膝, 因此其所書寫的歷史既有偏見又不公正。本文探討劉知幾 對《魏書》的批評當中所指出的書寫形式上和意識形態上的特點。劉知幾 的評議凸顯政權正統性的問題, 特別是南北政權對峙的問題, 事實上大大 地左右了該史學家對六朝 (220-589) 的歷史作品的評價。

* Damien Chaussende, post-doctorant au Centre de recherche sur les civilisations de l'Asie orientale (UMR 8155), remercie vivement François Martin pour ses précieuses suggestions. Ses remerciements vont également à Alexis Lycas et à Claude Chevaleyre. 


\section{Damien Chaussende}

Wei Shou 魏收 (506-572) acheva la première version de son Livre des Wei (Weishu 魏書) en 554, date à laquelle il le présenta à son commanditaire, l'empereur Wenxuan 文宣 (Gao Yang 高洋, r. 550-559) des Qi du Nord. Le contenu de l'ouvrage déclencha immédiatement des réactions si violentes dans l'aristocratie que le monarque demanda à l'auteur de reprendre son texte. De corrections en ajouts, le Livre des Wei acquit sa forme définitive après le règne de l'empereur Wucheng 武成 (Gao Zhan 高湛, r. 561-565). Son auteur mourut quelques années plus tard, et dès l'annexion des Qi par les Zhou en 577, des personnes, toujours blessées par certains passages de l'œuvre de Wei Shou, profanèrent la tombe de ce dernier : ils dispersèrent ses restes, condamnant ainsi les mânes de l'historien à errer jusqu'à la fin des temps ${ }^{1}$. Euvre contestée à bien des égards, le Livre des Wei n'en demeura pas moins l'histoire officielle des Wei du Nord et le premier ouvrage de cette importance consacré à une dynastie non-chinoise. Des tentatives de réécritures furent certes lancées par des souverains postérieurs, mais les ouvrages rédigés ne remplacèrent jamais totalement l'œuvre de Wei Shou. Elle est encore, à l'heure actuelle, la source textuelle la plus complète pour l'histoire de cette dynastie ${ }^{2}$.

Pour comprendre quelles sont les spécificités de l'ouvrage de Wei Shou et les raisons pour lesquelles il a toujours exhalé un parfum de scandale, il est utile de se tourner vers l'un de ses détracteurs les plus virulents, Liu Zhiji 劉知幾 (661-721) ${ }^{3}$, auteur du Traité de l'historien parfait (Shi-

${ }^{1}$ Bei Qishu 北齊書, Beijing : Zhonghua shuju, 1972, juan 37, p. 495.

${ }^{2}$ Sur la réception du Livre des Wei, $c f$. Jennifer Holmgren, "Northern Wei as a Conquest Dynasty: Current Perceptions ; Past Scholarship", Papers on Far Eastern History, 1989-40, p. 1-50. Notons que le Livre des Wei s'est mal transmis : sur les 114 chapitres que compte l'ouvrage (130 si l'on prend en compte les chapitres dédoublés en shang 上 et xia 下), 88 sont de Wei Shou ; les autres furent perdus totalement ou partiellement et restitués sous les Song principalement à partir des chapitres correspondants dans l'Histoire des dynasties du Nord (Beishi 北史). Ces chapitres restitués sont clairement indiqués dans l'édition de la Zhonghua shuju.

${ }^{3}$ Pour une présentation synthétique de Liu Zhiji et de son œuvre, $c f$. le travail pionnier de Guy Gagnon, La Notion d'histoire chez Liu Zhiji (661-721), fonctionnaire-historien à l'époque des Tang, thèse de doctorat, Paris : université Paris VII, 1988, qui a inspiré les traductions du présent article, en particulier celles des ter- 
tong 史通) ${ }^{4}$. Liu Zhiji fut lui-même un historien, mais c'est en tant que critique qu'il est passé à la postérité. Passionné par l'histoire dès son plus jeune âge, docteur (jinshi 進士) en 680, il entama une carrière officielle essentiellement tournée vers la tenue d'archives, la conservation d'ouvrages et surtout les activités d'historiographe, au sein du bureau de l'Histoire (Shiguan 史館), où il participa à la compilation de nombreux ouvrages historiques officiels, dont plusieurs annales véridiques (shilu 實錄). En 708, il démissionna brutalement de ses fonctions au bureau de l'Histoire, estimant les conditions de travail intolérables. Il envoya une lettre à ses supérieurs pour expliquer ses raisons et exposer un certain nombre de dysfonctionnements qui lui paraissaient insupportables. Cette lettre, qui forme le dernier chapitre de son traité ${ }^{5}$, ainsi que son autobiographie, également intégrée dans le Traité ${ }^{6}$, manifestent sa frustration ; en substance il affirme qu'au sein des institutions officielles où il a exercé ses fonctions d'historiographe, l'histoire ne s'écrit pas telle qu'elle devrait s'écrire. Liu Zhiji imagine alors la démarche de l'historien idéal. C'est ce qui le pousse à mettre en forme les nombreuses notes de lecture qu'il a prises au long

mes techniques propres à l'historiographie. Voir également Masui Tsuneo, "Liu Chih-chi 劉知幾 and the Shih-t'ung 史通”, Memoirs of the Research Department of the Toyo Bunko (The Oriental Library), 1976-34, p. 113-162 et Edwin Pulleyblank, "Chinese Historical Criticism: Liu Chih-chi and Ssu-ma Kuang", in William Beasley and Edwin Pulleyblank (ed.), Historians of China and Japan, Oxford: Oxford University Press, 1961, p. 135-166.

${ }^{4}$ J'emprunte cette traduction du titre du Shitong à Guy Gagnon, « La postface personnelle de Liu Zhiji au Shitong : un essai d'egohistoire ", in Jean-Pierre Drège (dir.), De Dunhuang au Japon, Paris : Droz, 1996, p. 337.

${ }^{5}$ 《À l'encontre du temps »wushi 忤時 (chap. B.12). Ce chapitre est intégralement traduit dans William Hung, "A T'ang Historiographer Letter of Resignation”, Harvard Journal of Asiatic Studies, 1969-29, p. 5-52. Les références au Shitong se présenteront désormais ainsi : la première partie (neipian 內篇) sera désignée par A, la seconde (waipian 外篇) par B ; la lettre sera suivie du numéro du chapitre (pian 篇), puis du numéro de la page dans l'édition de Pu Qilong 浦起 龍, Shitong tongshi 史通通釋 (abrégé en STTS), Shanghai : Shanghai guji, 2009, par exemple : STTS, B.2.339.

${ }^{6}$ Il s'agit de la «postface personnelle » (zixu 自敘, chap. A.36), traduite intégralement dans Guy Gagnon, « La postface personnelle de Liu Zhiji au Shitong : un essai d'egohistoire ». 
des années pour en tirer le Traité de l'historien parfait, mis en circulation deux ans après sa démission. Ce texte est l'occasion pour Liu Zhiji de transmettre ses idées en matière d'historiographie, tant du point de vue théorique que du point de vue pratique. En prenant appui sur les œuvres du passé, qu'il commente, compare et évalue, il livre ses réflexions sur le statut et la finalité de l'histoire et dispense ses leçons sur la manière dont on doit l'écrire. À maints égards, il préfigure la critique philologique du mouvement kaozheng 考證 des lettrés Qing, en particulier par son attitude souvent iconoclaste et, sur bien des points, très critique face aux textes qu'il se propose d'examiner.

Le Livre des Wei est, dans le Traité, un cas vraiment à part : il y apparaît comme le repoussoir de Liu Zhiji. Celui-ci ne manque en effet aucune occasion de citer Wei Shou comme le contre-modèle de l'historien idéal : en plus d'être un plagiaire et un historien corrompu, Wei Shou se montrerait partial, jaloux, rancunier, injuste, et s'écarterait excessivement des normes historiographiques en usage. Qu'ils portent sur le fond, sur la forme, ou sur l'homme lui-même, les commentaires de Liu Zhiji sont extrêmement précieux car ils dévoilent maints traits spécifiques à Wei Shou et permettent surtout de cerner ex negativo son projet historiographique. Le présent article ne prétend pas livrer une étude approfondie du Livre des Wei, dont la longueur et la richesse le rendent comparable aux Mémoires historiques (Shiji 史記) de Sima Qian 司馬遷 7. Je tenterai plus simplement de saisir les particularités essentielles du Livre des Wei telles que les a mises en lumière Liu Zhiji.

Commençons par voir de manière synthétique l'ensemble de ces critiques.

\footnotetext{
${ }^{7} \mathrm{Il}$ n'existe pas de telle étude à ma connaissance, on peut néanmoins se reporter au travail synthétique de Zhou Yiliang 周一良, 《Wei Shou zhi shixue 》魏收之 史學 (L'historiographie de Wei Shou), in Wei Jin Nanbeichao shi lunji 魏晉南 北朝史論集 (Études sur l'histoire des Wei, des Jin et des dynasties du Sud et du Nord), Beijing : Zhonghua shuju, 1963, p. 236-272.
} 


\section{Le Livre des Wei : une «saleté d'histoire" ?}

La lecture du Livre des Wei a beaucoup marqué Liu Zhiji. Dans le Traité, Wei Shou et son ouvrage apparaissent en effet aussi souvent que la Monographie des Trois Royaumes (Sanguozhi 三國志) de Chen Shou 陳壽, l'un des fleurons de la littérature historique, et plus que le Livre des Han postérieurs (Hou Hanshu 後漢書) de Fan Ye 范曄, le Livre des Song (Songshu 宋書) de Shen Yue 沈約 ou encore le Livre des Qi du Sud (Nan Qishu 南齊書) de Xiao Zixian 蕭子顯 ${ }^{8}$. Le Livre des Wei est certes beaucoup moins fréquemment mentionné que les Mémoires historiques de Sima Qian ou le Livre des Han (Hanshu 漢書) de Ban Gu 班固 - de loin les deux histoires de la forme annales-biographies (jizhuan $t i$ 紀傳體) les plus citées dans le Traité ${ }^{9}$ - mais il n'en demeure pas moins un ouvrage dont Liu Zhiji parle beaucoup, et, nous allons le voir, surtout en mal.

Le plus long développement consacré au Livre des Wei consiste en une notice synthétique du chapitre B.2, «Histoires officielles du passé et du présent »(Jingu zhengshi 古今正史) ${ }^{10}$. Elle se divise en deux sections : la première expose l'histoire de la compilation du texte ; la seconde, après quelques jugements généraux sur l'œuvre, relate le scandale qu'elle suscita et les révisions successives qui y furent apportées :

Dans la deuxième année de l'ère Tianbao des Qi (551), [l'empereur Gao Yang] ordonna à Wei Shou, Inspecteur de la Bibliothèque impériale, de procéder à une large collecte des documents historiques [concernant la dynastie des Wei] afin d'en compiler l'histoire. Il ordonna également à Diao Rou 习柔, Xin Yuanzhi 辛元植, Fang Yanyou 房延祐, Mu Zhongrang 睦仲讓, Pei Angzhi 裴昂之, Gao Xiaogan 高孝幹 et d'autres d'aider à

${ }^{8}$ D'après les index de l'édition de Nishiwaki Tsuneki 西劦常記, Shitsū gaihen 史通外篇, Tokyo : Tōkai daigaku shuppankai, 2002 et Shitsū naihen 史通內篇, Tokyo : Tōkai daigaku shuppankai, 1989 ; le Shitong dans son ensemble (neipian et waipian) contient 33 occurrences du nom Wei Shou et 27 du titre Weishu, contre 32 et 31 pour le Sanguozhi et Chen Shou, 24/28 pour Songshu /Shen Yue, 21/24 pour Hou Hanshu/Fan Ye, 8/13 pour Nan Qishu/Xiao Zixian.

${ }^{9}$ Dans le waipian, Nishiwaki recense 38 mentions du nom Sima Qian et $40 \mathrm{du}$ titre Shiji ainsi que 48 Ban Gu et 47 Hanshu. Les mentions des deux ouvrages et de leurs auteurs sont si nombreuses dans le neipian que le traducteur japonais les a omises dans les index.

${ }^{10}$ STTS, B.2.339. 


\section{Damien Chaussende}

l'élaboration de l'ouvrage. Wei Shou craignait que les historiens sous son autorité ne lui manquent d'égard. Diao, Xin et les autres n'avaient aucun talent pour l'histoire. C'est seulement parce qu'ils présentaient les apparences de l'érudition qu'on les affecta à son service. Par la suite, [l'équipe] rechercha les généalogies des familles, en fit une sélection raisonnée et compila le Livre des Wei. Ce dernier [traite de la période allant] de l'empereur Daowu 道武 (r. 386-409) à l'empereur Xiaojing 孝靖 (=Xiaojing 孝 靜, Yuan Shanjian 元善見, $r$. 534-550). Ses annales, ses biographies et ses monographies forment en tout cent trente chapitres.

Shou flatte les Qi, et se montre partial envers la maison impériale des Wei. Il prend parti pour les dynasties du Nord et calomnie à l'envi celles du Sud. Il détestait ceux qui l'avaient surpassé et se plaisait, par ailleurs, à ressasser de vieilles rancunes. Aussi injurie-t-il dans [son œuvre] tous les hommes vertueux et bien nés envers qui il éprouvait une rancœur, en dissimulant leurs bonnes actions. Sa haine le poussa à diffamer jusqu'aux trisaïeuls de ses contemporains. Lorsque l'ouvrage fut remis au trône, l'empereur ordonna à Shou d'en débattre avec les [membres] des familles [incriminées] au département des Affaires d'État. Plus d'une centaine de personnes s'étaient plaintes. À cette époque, le directeur du département des Affaires d'État, Yang Zunyan 楊遵彥, était l'un des ministres les plus en vue. Il était très écouté de la cour. Wei Shou avait, dans son ouvrage, rédigé des biographies particulièrement flatteuses pour les membres de son clan. Aussi, Yang Zunyan fut-il un précieux soutien pour l'historien. Ceux qui l'avaient critiqué furent sévèrement punis. Certains même finirent leurs jours en prison. Les médisances allèrent alors bon train. Sous le règne de l'empereur Xiaozhao 孝昭 (Gao Yan 高演, $r$. 560-561), on ordonna à Wei Shou de reprendre son travail pour qu'il puisse être publié officiellement. L'empereur Wucheng 武成 (Gao Zhan), quant à lui, demanda l'avis de ses ministres concernant l'ouvrage. Ils déclarèrent que son contenu n'était toujours pas conforme à la réalité. Le souverain ordonna à nouveau une révision ; elle entraîna une modification substantielle de l'ouvrage. En raison de cela, le Livre des Wei fut peu apprécié de ses contemporains, qui le qualifièrent de « saleté d'histoire ». ${ }^{11}$

Cette notice guère flatteuse donne globalement le ton de 1'ensemble du discours de Liu Zhiji sur le Livre des Wei : Wei Shou est partial puisqu'il « flatte la maison des Qi » et « prend le parti des dynasties du Nord tout en calomniant à l'envi celles du Sud »; il se sert en outre de son

${ }^{11}$ STTS, B.2.339. 
œuvre pour régler des comptes personnels. En conclusion de la notice, Liu Zhiji ne manque pas de rappeler le sobriquet que reçut le Livre des Wei, une « saleté d'histoire» (huishi 穢史).

Les commentaires de Liu Zhiji sur Wei Shou ne surgissent pas ex abrupto, mais s'inscrivent dans une continuité de critiques qui commençèrent dès la publication du Livre des Wei. Wei Shou avait été attaqué de son vivant, mais il s'agissait de personnes dont les familles étaient mentionnées dans l'ouvrage, qui, à l'époque de sa publication, était en grande partie une œuvre d'histoire contemporaine. Le détail des accusations portées contre Wei Shou se trouve dans les deux biographies qui lui furent consacrées, l'une se trouvant dans Livre des Qi du Nord (Bei Qishu 北齊 書) de Li Baiyao 李百藥 (564-647) et l'autre dans l'Histoire des dynasties $d u$ Nord (Beishi 北史) de Li Yanshou 李延壽 ( $f$. 650) ${ }^{12}$. À vrai dire, les deux textes sont très proches et pour certains passages, identiques au mot près. C'est que le Livre des Qi du Nord s'est mal transmis, et dans les éditions Song - les plus anciennes que nous possédions - certains chapitres, parmi lesquels la biographie de Wei Shou, ont été restitués à partir des parties correspondantes de l'Histoire des dynasties du Nord ${ }^{13}$.

Le récit du scandale ainsi que le qualificatif de « saleté d'histoire » ${ }^{14}$ figurent dans les deux biographies. Ces dernières conservent également la trace de plaidoyers en faveur du texte de Wei Shou : des défenseurs n'hésitent pas à dire que « le Livre des Wei de Wei Shou est une œuvre érudite composée avec un immense talent, que c'est un grand accomplissement pour la Maison de Wei » et « un ouvrage définitif qui se transmettra pour l'éternité $\gg{ }^{15}$.

Le souverain fondateur des Sui, l'empereur Wen 文 (r. 581-604), considérant que le Livre des Wei de Wei Shou n'était pas satisfaisant, commanda une nouvelle version à un certain Wei Dan 魏澹 $(580-645){ }^{16}$. De

${ }^{12}$ Bei Qishu, juan 37, p. 483-495, Beishi 北史, Beijing : Zhonghua shuju, 1974, juan 56, p. 2023-2038.

${ }^{13}$ Voir la note des éditeurs de la Zhonghua shuju dans Bei Qishu, juan 37, p. 496, note 1 .

${ }^{14}$ Beishi, juan 56, p. 2032 ; Bei Qishu, juan 37, p. 489.

${ }^{15}$ STTS, B.2.339.

${ }^{16}$ Suishu, juan 58, p. 1417 et STTS, B.2.339. Sur Wei Dan et son histoire, $c f$. 


\section{Damien Chaussende}

fait, la démarche était tout à fait cohérente d'un point de vue politique : les Sui tiraient leur légitimité des Zhou, qui tiraient la leur des Wei occidentaux. Il fallait donc réécrire l'histoire des Wei en délégitimant les Wei orientaux ( $c f$. plus bas, en particulier le diagramme). Cette histoire ne nous a pas été transmise ; il n'en reste que quelques fragments cités ici et là. Cependant, la biographie de Wei Dan du Livre des Sui (Suishu 隋書) contient un document dans lequel l'historien expose ses principes historiographiques et critique au passage Wei Shou ${ }^{17}$. On trouve dans ce texte quelques thèmes qui seront repris et amplifiés par Liu Zhiji, par exemple l'idée que les Wei provenaient à l'origine de tribus barbares des confins nordiques et qu'il n'était pas utile de consacrer beaucoup de place à cette préhistoire peu intéressante, comme le fait Wei Shou au début de son ouvrage ; ou encore les questions d'appellations des souverains, un enjeu historiographique capital puisque la désignation même d'un monarque (nom personnel, nom posthume ou nom de temple) manifeste le degré de légitimité que l'historien lui accorde.

Lorsqu'il fut question, au début des Tang, de compléter ou de réécrire l'histoire de certaines dynasties du Nord et du Sud, le cas des Wei fut discuté. Finalement, l'histoire n'en fut pas réécrite car, rapporte l'Ancien livre des Tang (Jiu Tangshu 舊唐書), « les conseillers considéraient que, pour l'histoire des Wei, on disposait déjà des deux ouvrages de Wei Shou et de Wei Dan, qui étaient complets et détaillés. Il n'en fut donc pas composé de nouveau $"{ }^{18}$. À cette époque, donc, le Livre des Wei de Wei Shou fut jugé satisfaisant et complémentaire de celui de Wei Dan, à la différence de la dizaine de Livres des Jin (Jinshu 育書) disponibles au début des Tang : l'empereur Taizong 太宗 (r. 626-649) avait en effet ordonné à une équipe d'historiens la composition d'une nouvelle histoire des Jin qui remplacerait toutes celles existantes.

Li Yanshou, dans son Histoire des dynasties du Nord, qu'il présente au trône en 659, semble être lui aussi un partisan du travail de Wei Shou, qu'il considère dans l'ensemble satisfaisant. En effet, à la fin de la biogra-

Albert Dien, "Wei Tan and the Historiography of the Wei-shu", in Paul Kroll, David Knechtges (ed.), Studies in Early Medieval Chinese Literature and Culture History, Provo: T'ang Study Society, 2003, p. 399-466.

${ }^{17}$ Suishu, juan 58, p. 1417-1429; l'ensemble de la biographie de Wei Dan, et donc ce document, est intégralement traduite dans Albert Dien, opus cit.

${ }^{18}$ Jiu Tangshu 舊唐書, Beijing : Zhonghua shuju, 1975, juan 73, p. 2598. 
phie de ce dernier, il rédige un jugement récapitulatif (lun 論) relativement positif ${ }^{19}$ :

[Wei Shou] compila un ouvrage sur les Wei dans la lignée [de ceux composés] par Ban Gu et Sima Qian. Son style est plein de grâces mais respecte les règles; son œuvre est riche sans être confuse. Dans ses jugements et ses avertissements, [Wei Shou] sonde les profondeurs et approche les lointains (c.-à-d. qu'il est clairvoyant). Son seul objectif étant de préserver la vérité des faits, il se plait à affronter les secrets. Il ne dit absolument rien de sa propre famille, ni de celles dont il était l'intime. Voilà bien les seules injustices dont on peut le charger. ${ }^{20}$

On peut penser qu'au milieu du vII siècle, $l^{\text {' }}$ ' affaire Wei Shou » paraît refroidie ; les dynasties du Nord et du Sud, et les questions de légitimité afférentes, semblent n'être plus que de l'histoire ancienne. Li Yanshou publie en effet ses deux histoires (Nanshi et Beishi) dans un esprit de synthèse étonnant par rapport aux ouvrages sur lesquels il se fonde. Il consacre des annales impériales à tous les souverains des États ayant fait l'objet d'histoires officielles, c'est-à-dire les Song, Qi, Liang et Chen pour le Sud, et les Wei, Qi, Zhou et Sui pour le Nord. Pour ce qui est des Wei, il traite de manière égale les monarques des Wei occidentaux et ceux des Wei orientaux.

Dans son Traité, Liu Zhiji se place résolument dans la lignée des détracteurs du Livre des Wei et rouvre le dossier États du Sud versus États du Nord. C'est sur un terrain proche de celui de Wei Dan qu'il attaque Wei Shou : le terrain proprement historiographique et politique. Le scandale suscité par l'œuvre à sa parution ne l'intéresse que dans la mesure où il manifeste pour lui un trait de son auteur. Par ailleurs, la question de la légitimité des Wei s'articule moins dans l'antagonisme entre Wei orientaux et Wei occidentaux que dans la question États du Nord/États du Sud. C'est ce qui explique pourquoi Liu Zhiji renvoie dos à dos Wei Shou et Wei Dan, considérant que le second n'a pas livré un meilleur texte que le premier, « il a remplacé des erreurs par d'autres erreurs » (yi fei yi fei 以非易非) dit-il ${ }^{21}$.

\footnotetext{
${ }^{19}$ Notons qu'on ne trouve pas la contrepartie de ce jugement dans le Livre des Qi du Nord.

${ }^{20}$ Beishi, juan 56, p. 2048.

${ }^{21}$ STTS, B.9.481.
} 


\section{Damien Chaussende}

Mis à part la notice mentionnée plus haut, Liu Zhiji cite le Livre des Wei à d'autres reprises dans son traité, en particulier dans la première partie, composée de chapitres thématiques traitant des questions de structure, de rhétorique ou de méthodologie historique. Certaines appréciations sont relativement mineures et portent sur des points de détail. Par exemple, dans le chapitre A.8, « Les monographies » (shuzhi 書志), Liu Zhiji reproche à Wei Shou d'avoir innové en rédigeant un traité consacré au bouddhisme et au taoïsme ${ }^{22}$, et juge en revanche excellent le choix de n'avoir traité que des Wei du Nord dans la monographie consacrée à l'astronomie ${ }^{23}$. De même, Liu Zhiji apprécie le placement de l'ensemble des monographies en fin d'ouvrage, et non entre les annales et les biographies, comme c'est le cas dans d'autres histoires ${ }^{24}$. Ce sont d'ailleurs les seuls passages où Liu Zhiji valorise le Livre des Wei.

D'autres critiques sont plus importantes, soit parce qu'elles portent sur l'ouvrage dans son ensemble, soit parce qu'elles sont répétées plusieurs fois dans le traité, soit encore parce que Liu Zhiji prend la peine d'étayer son propos par des exemples précis. Ce sont ces jugements qui révèlent le mieux le point de vue du critique sur Wei Shou et qui mettent en lumière les traits fondamentaux du projet historiographique de ce dernier. Ces attaques sont dispersées dans le traité et apparaissent dans les chapitres thématiques concernés, ce qui amène parfois certaines redites.

Ces critiques, que nous allons explorer au long de cet article, se rattachent globalement aux trois grandes thématiques suivantes : 1. pour Liu Zhiji, Wei Shou excède les limites temporelles et spatiales de son sujet, qui doit être de traiter des Wei, de leur fondation en tant qu'entité impériale jusqu'à leur chute ; 2. prenant le parti des Qi du Nord, son traitement de l'histoire des Wei est excessivement pro-Gao et de ce fait délégitime la cour des Wei orientaux ; 3. enfin, Wei Shou est trop hostile aux pouvoirs politiques installés dans le Sud.

Ces lignes de force qui sous-tendent l'histoire de Wei Shou sont la manifestation presque directe des contraintes politiques qu'il subissait en tant

${ }^{22}$ STTS, A.8.52.

${ }^{23}$ STTS, A.8.54. Wei Shou se restreint aux Wei, par opposition à d'autres histoires comme le Livre des Han de Ban Gu, qui embrasse trop large. Cf. STTS, A.8.53.

${ }^{24}$ STTS, A.13.96. 
qu'historien officiel, car, comme je l'ai indiqué plus haut, son ouvrage ne fut pas une initiative privée, mais fut commandité par le pouvoir politique en place.

\section{Les contraintes d'une histoire des Wei composée sous les Qi}

La lèse-majesté historiographique existe bel et bien dans la Chine classique, et nombreux furent les historiens à avoir subi la colère de leur prince pour quelques mots de trop ${ }^{25}$. Liu Zhiji, qui a conscience de cet état de fait ${ }^{26}$, considère néanmoins que l'historien idéal doit savoir prendre ses distances avec son souverain et s'affranchir au maximum des contraintes. Wei Shou est à ses yeux l'archétype du lettré servile envers le pouvoir politique qui l'employait. Lorsque Liu Zhiji en vient à critiquer ce travers chez un autre, c'est à Wei Shou qu'il le compare :

Dans ses annales et ses biographies, [Xu] Jingzong 敬宗 ${ }^{27}$, lorsqu'il ne déforme pas les faits pour répondre à un ordre de son souverain, les maquille en fonction de ses haines personnelles. Ses critiques et ses louanges n'ont aucun fondement. Il est à Wei Shou ce que Cai Yong 蔡握 était à Zhang Heng 張衡 ! ${ }^{28}$

Un historien n'écrit pas pour faire plaisir à son prince, nous dit Liu

${ }^{25}$ J'ai traité cette question dans une communication intitulée « Être historien dans la Chine classique : un métier à risque », à paraître prochainement dans les actes du colloque « La Faute et sa punition dans les civilisations orientales » (Institut du Proche-Orient ancien du Collège de France, Société asiatique, Paris, 21-22 juin 2010) aux Cahiers de l'Institut du Proche-Orient ancien.

${ }^{26} C f$. par exemple STTS, A.24.179 : «Si l'on écrit avec droiture à une époque où cela est possible, tout se passe bien. Lorsqu'on le fait à une époque où il n'est pas permis de le faire, il y a péril. »

${ }^{27} \mathrm{Il}$ est ici question d'une Histoire nationale (guoshi 國史, c'est-à-dire une histoire de la dynastie en cours) des Tang composée sous la direction de Xu Jingzong 許 敬宗 (592-672), grand ministre sous le règne de Gaozong 高宗 (r. 649-683), et partisan de la première heure de Wu Zetian 武則天 ( $r$. 684-705).

${ }^{28}$ Zhang Heng mourut alors que la mère de Cai Yong était enceinte. Comme les deux étaient semblables dans leurs traits et leurs talents, on disait que le second était la réincarnation du premier. STTS, B.2.347. 
Zhiji ; il doit même prendre le risque de lui déplaire si besoin est. Le chapitre A.24, «Écrire avec droiture » ( $z h i s h u$ 直書), est entièrement consacré à ce thème et mentionne quelques historiens martyrs célèbres. Est évoqué, entre autres, Cui Hao 崔浩 (m. 450) ${ }^{29}$, qui fut condamné à mort par l'empereur Taiwu 太武 (r.423-452) pour avoir composé une histoire des Wei que le souverain jugea infamante pour sa dynastie ${ }^{30}$. C'est que Cui Hao, un Chinois, n'hésita pas à insister sur les origines steppiques des Tuoba et à relater quelques échecs militaires cuisants. Ce n'est pas le cas de l'œuvre de Wei Shou, qui, au contraire, met en valeur les Wei depuis leur « préhistoire », c'est-à-dire depuis le Dai 代, une chefferie Tuoba de l'époque des Jin. Aussi, non seulement Liu Zhiji reproche-t-il à Wei Shou d'avoir falsifié l'histoire au profit des Wei et des Qi, mais il blâme en outre Gao Yang, le commanditaire de l'œuvre, pour avoir avoir soutenu son historien en étant parfaitement conscient des imperfections du Livre des Wei:

La fonction de l'historien est de noter les mérites et les fautes, de mettre à l'honneur les vertus et d'abaisser les vices, de transmettre pour l'éternité aussi bien la gloire des succès que la honte des défaites. Si l'on transgresse ces règles, est-on bien digne de sa charge ? Depuis l'Antiquité, c'est en raison de leur probité que certains [historiens] ont été punis ; la falsification, elle, n'attire jamais les châtiments. [...] C'est pour cela que Xuan de Qi 齊 宣 (Gao Yang) n'a pas désapprouvé Wei Shou et son histoire des Wei, bien qu'elle fût partiale. [Ce souverain] a permis à son historien de se laisser aller à ses haines et ses sympathies et de déterminer les vices et les vertus de manière arbitraire. ${ }^{31}$

Si Gao Yang et après lui ses successeurs ont soutenu Wei Shou - en particulier face à ceux qui s'étaient plaints de l'œuvre - c'est qu'à leurs yeux, l'historien avait admirablement rempli son rôle. Et ce n'était pas tâche facile, car les contraintes politiques étaient nombreuses.

${ }^{29}$ STTS, A.24.179.

${ }^{30}$ L'affaire est racontée dans les biographies de Cui Hao : Beishi, juan 21, p. 789 et Weishu魏書, Beijing : Zhonghua shuju, 1974, juan 35, p. 825-826. Une version synthétique apparaît dans Zizhi tongjian 資治通鑑, Beijing : Zhonghua shuju, 1956, juan 125, p. 3941-3944.

${ }^{31}$ STTS, A.25.185. 
Au long de sa vie, Wei Shou a traversé en effet une période très instable politiquement ${ }^{32}$; il s'en est tiré principalement grâce à sa plume. Très expérimenté dans la composition de documents officiels du type décret ou proclamation, il se vit plusieurs fois confier la rédaction d'annales historiques. Son talent littéraire éclot très tôt et il devint dans sa jeunesse Érudit à l'université impériale (Taixue boshi 太學博士). Il échappa au massacre perpétré par Erzhu Rong 爾朱榮 (493-530) en 528, et, en 531, se vit nommé annaliste en charge des chroniques impériales (qijuzhu 起居注). La situation à la cour était à cette époque extrêmement troublée. De nombreux ministres et chefs de guerre se disputaient le pouvoir et pas moins de cinq empereurs fantoches se succédèrent sur le trône entre 529 et 534 . Wei Shou parvint malgré tout à tirer son épingle du jeu. En 534, un condottiere, Gao Huan 高 歡 (496-547), s'installa à Ye 鄴 et plaça Yuan Shanjian 元善見 (524-552, r. 534-550) sur le trône, tandis que le souverain en titre, Yuan Xiu 元修 (510-535, r. 532-535), se réfugiait auprès de Yuwen Tai 宇文泰 (507-556), un autre «maire du palais », à Chang'an 長安. Il y eut donc dès lors deux empereurs des Wei, l'un régnant à Chang'an, sur les Wei dits occidentaux, l'autre à Ye, sur les Wei orientaux ( $c f$. diagramme plus bas). Wei Shou prit le parti de Gao Huan et le suivit à Ye, où d'honneurs en honneurs, il poursuivit sa carrière. On le confirma dans ses fonctions d'historiographe officiel et on l'envoya même en ambassade à Liang, dans le Sud, en 537. En 550, Gao Yang, fils de Gao Huan, procéda au changement dynastique : il mit un terme aux Wei orientaux et fonda les Qi du Nord en recevant l'abdication de Yuan Shanjian. Il chargea Wei Shou de rédiger tous les documents officiels relatifs à la transition dynastique ${ }^{33}$. Une fois sur le trône, il commanda une histoire des Wei à Wei Shou. Ce dernier s'exécuta et présenta l'ouvrage au trône en 554, avec les conséquences que l'on sait.

${ }^{32}$ Pour une présentation générale des événements de la période, en particulier pour la guerre civile des années 520-530, Étienne Balazs, «Études sur la société et l'économie de la Chine médiévale. I. Le traité économique du "Souei-Chou" ", T'oung Pao, 1953-42 (3/4), p. 241-262.

${ }_{33}$ Bei Qishu, juan 37, p. 487 et Beishi, juan 56, p. 2030. D'après Bei Qishu, juan 30, p. 408, Wei Shou fut chargé de rédiger le Texte des Neuf distinctions (jiuxiwen 九錫文, conservé dans Bei Qishu, juan 4, p. 45-47), l'acte d'abdication (shanrang zhaoce 禪讓詔策, Bei Qishu, juan 4, p. 47-49), la supplique des conseillers (quanjin wenbiao 勸進文表, mentionnée en Bei Qishu, juan 4, p. 49) ainsi que la proclamation inaugurale (jiantai 建臺, Bei Qishu, juan 4, p. 49-50). 
La date à laquelle est commanditée l'histoire des Wei n'est pas anodine. C'est en 551, soit un an après avoir fondé les Qi du Nord, que Gao Yang ordonne à Wei Shou de rédiger l'ouvrage. La proximité temporelle entre cet ordre et le changement dynastique qui vient d'avoir lieu est particulièrement éloquente : Gao Yang veut montrer qu'une ère est révolue et que les Wei appartiennent au passé - alors qu'à Chang'an un autre pouvoir politique continue à se clamer le continuateur des Wei. Aussi, Wei Shou a-t-il non seulement contribué à la transition dynastique - puisqu'il a été chargé d'en rédiger les textes officiels - mais il est aussi en quelque sorte le fossoyeur historiographique de la dynastie déchue.

L'histoire qu'il rédige est on ne peut plus marquée politiquement. Elle est un véritable instrument politique et, si elle fut agréée par le souverain commanditaire (malgré les corrections qui lui furent demandées), c'est qu'elle répondait de manière satisfaisante à plusieurs contraintes idéologiques que Liu Zhiji n'a pas manqué de remarquer.

\section{Les États du Nord de 399 à 617}

Wei du Nord 北魏 (399-534)

clan Tuoba (Tabgatch) 拓拔 puis Yuan 元 ${ }^{34}$

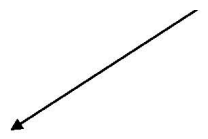

Wei occidentaux 西魏 - Chang'an

(534-557)

(clan Yuwen 宇文)

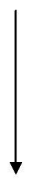

Zhou du Nord 北周 (557-581)

Sui 隋 (581-618)

Tang 唐 (618-907)

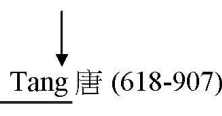

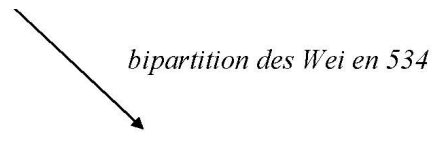

Wei orientaux 東魏 - Ye

(534-550)

(clan Gao 高)

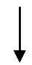

Qi du Nord 北齊 (550-577)

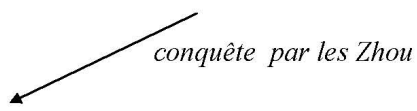

${ }^{34}$ Le clan Tuoba adopte le patronyme chinois de Yuan en 495, sur décision de l'empereur Xiaowen 孝文 (r. 471-499). 
En effet, les Qi du Nord tirant leur légitimité politique des Wei orientaux (puisqu'ils leur succèdent en recevant l'abdication « volontaire » du souverain de la dynastie précédente ${ }^{35}$ ), il s'ensuit que dans le Livre des Wei de Wei Shou:

1. l'État légitime est celui des Wei orientaux, si bien que

2. les Wei occidentaux sont illégitimes, et que

3. sont également illégitimes les autres pouvoirs politiques établis sur l'écoumène chinois (en particulier les États du Sud, tel que les Jin orientaux, les Song, les Qi et les Liang) ${ }^{36}$.

4. Les Gao, famille impériale des Qi du Nord, ayant joué un rôle significatif sous les Wei, ne pourront être présentés que de manière positive.

Or, un désaccord inconciliable oppose Wei Shou et Liu Zhiji : pour ce dernier, c'est dans les États du Sud que résidait la légitimité.

\section{Nord contre Sud}

En effet, Liu Zhiji affirme clairement dans son traité que, pendant la période de division territoriale (de la chute des Jin occidentaux jusqu'à la réunification des Sui), seul le fils du Ciel établi à Nankin est le véritable dépositaire du mandat céleste :

Dès lors que Chang'an et Luoyang 咸洛 ne furent plus défendues ${ }^{37}$, les tortues et les tripodes se déplacèrent au Sud : la rive méridionale du fleuve Bleu devint la patrie des rites et de la musique ; Jinling 金陵 (c'està-dire Nankin), le conservatoire des livres et des écrits. C'est pourquoi se

\footnotetext{
${ }^{35}$ Sur ce mode de transition dynastique, $c f$. François Martin, « Des faux qui ne trompent personne - Les textes d'abdication sous les Six Dynasties ", ExtrêmeOrient, Extrême-Occident, 2010-32, p. 13-39. Pour une étude de cas, $c f$. Damien Chaussende, Des Trois Royaumes aux Jin. Légitimation du pouvoir impérial en Chine au III siècle, Paris : Les Belles Lettres, 2010.

${ }^{36}$ Dans la philosophie politique chinoise classique, le Ciel ne délivre qu'un seul mandat : il ne saurait donc y avoir deux fils du Ciel.

${ }^{37}$ Sous les Jin occidentaux, Luoyang, la capitale, est conquise par les Xiongnu et leurs alliés en 311 ; Chang'an, la seconde capitale, tombe en 316.
} 


\section{Damien Chaussende}

sont maintenus là-bas un langage modéré, des usages anciens et un art du discours empli de noble élégance, et que, malgré les troubles et les affaires pressantes ${ }^{38}$, les livres n’ont pas été oubliés.

Les États du Sud sont non seulement les seuls à être régis par un souverain légitime (car il possède « les tortues et les tripodes » 龜鼎, symboles des cérémonies officielles et des rites divinatoires), mais ce sont aussi les conservatoires de la culture ancienne. Il en va tout autrement des États du Nord, à demi sauvages pour Liu Zhiji :

Les pays du centre ${ }^{39}$ 中國 n'étaient pas ainsi. Pourquoi ? Parce qu'à cette époque, la patrie des anciens souverains était tombée aux mains des barbares Man 蠻 et Mo 腼. Ces gens, qui ont les cheveux épars et le pan de tunique rabattu à gauche ${ }^{40}$, occupaient les provinces divines 神州. On pouvait, à l'occasion, y rencontrer des personnes qui débattaient à la manière de Juzhi 駒支 ou qui étaient aussi instruites que Tanzi 邩子, mais c'était assez rare ${ }^{41}$. Aussi, Yanluan 彥鸞 (Cui Hong 崔鴻, fl. ca 520), dans ses histoires des pays illégitimes, Wei Shou, dans son Livre des Wei, ou encore Hong 弘 (Niu Hong 牛弘), dans son Livre des Zhou, furent contraints de dissimuler les langues barbares et de les transposer en langue chinoise, tout comme Yang You 楊由 avec ses moineaux et Ge le Jie 介葛 avec son buffle ${ }^{42}$. Faire ainsi, passe encore. Mais dans leurs écrits, ces historiens

${ }^{38}$ Les « troubles et les affaires pressantes » (dianpei zaoci 顛沛造次) sont une allusion aux Entretiens de Confucius, IV.5 : «Si l'homme de bien abandonne la bienveillance, comment soutiendra-t-il son titre d'"homme de bien"? L'homme de bien ne l'abandonne jamais, pas même le temps d'un repas. Il y demeure toujours, même au milieu des affaires les plus pressantes, même au milieu des plus grands troubles. »

${ }^{39} \mathrm{C}$ 'est à dire ceux qui occupaient le bassin de la Wei et la grande plaine du Nord. Les « provinces divines » 神州 en sont un synonyme assez fréquent.

${ }^{40}$ «Les cheveux épars et le pan de tunique rabattu à gauche» (pifa zuoren 被發左 衽) : c'est ainsi que Confucius décrit les barbares dans un passage des Entretiens (XIV.17).

${ }^{41}$ Juzhi est un chef non-chinois mentionné dans le Zuozhuan, cité ici comme un exemple de bon orateur. Tanzi, également mentionné dans le Zuozhuan, est un prince étranger que Confucius prit pour maître en raison de sa sagesse.

${ }^{42}$ Yang You vécut sous les Han postérieurs et se rendit célèbre pour avoir correctement interprété la présence de moineaux posés sur un bâtiment officiel. Ge, chef des Jie, mentionné dans le Zuozhuan, s'illustra quant à lui pour avoir 
brodent sans aucun fondement et embellissent inutilement; ils invoquent le Classique de la poésie, le Classique des documents, et prennent pour modèle les Mémoires historiques et le Livre des Han. Ainsi, les Juqu 沮渠 et les Qifu 乞伏 ${ }^{43}$ sont dépeints comme des lettrés de l'ère Yuanfeng [de l'empereur Wu des Han]. Les Tuoba 拓跋 et les Yuwen 宇文, quant à eux, se mettent à parler aussi bien que pendant l'ère Zhengshi [des Cao-Wei]. Enjoliver les choses à ce point, je ne vois rien de pire à cela ! ${ }^{44}$

Selon le Nouveau livre des Tang (Xin Tangshu 新唐書) ${ }^{45}$, Liu Zhiji appartient au clan des Liu de Pengcheng 彭城, le même que le clan impérial des Liu-Song. C'est peut-être ce qui explique pourquoi Liu Zhiji est tant attaché à la légitimité des États du Sud ${ }^{46}$. Cette question revient à plusieurs reprises dans le Traité ${ }^{47}$, et oriente considérablement le jugement du critique sur Wei Shou, qui devient pour lui le type même de l'historien qui non seulement n'a pas su discerner où était la légitimité, mais qui en plus a défendu l'indéfendable : les souverains barbares. Si le critique ne remet pas en cause l'idée d'une histoire consacrée à des pouvoirs illégitimes, il ne lui plaît guère de voir dénigrés les États méridionaux au profit de territoires tombés aux mains de peuples étrangers. Dans le chapitre A.25, « La falsification » (qubi 曲筆), il revient sur le sujet - et donc aussi sur

interprété les mugissements d'un buffle.

${ }^{43}$ Les Juqu sont le clan fondateur des Liang septentrionaux 北涼, les Qufu, celui des Qin occidentaux 西秦. Ces deux États appartiennent aux Seize Royaumes traités par Cui Hong dans ses Printemps et automnes des Seize Royaumes (Shiliuguo chunqiu 十六國春秋), une œuvre qui ne nous a pas été transmise dans son intégralité.

${ }^{44}$ STTS, A.20.140.

${ }^{45}$ Cf. la biographie de Liu Yanyou 劉延祐, oncle de Liu Zhiji, dans Xin Tangshu 新唐書, Beijing : Zhonghua shuju, 1975, juan 201, p. 5732-5733. Celle de Liu Zhiji se trouve au juan 132, p. 4519.

${ }^{46}$ Cf. Jennifer Holmgren, "Northern Wei as a Conquest Dynasty", p. 6.

${ }^{47}$ Par exemple STTS, A.12.90 : « Dès lors que les Barbares eurent promulgué leurs lois, le pays se fragmenta en plusieurs maisons [régnantes]. Les dynasties du Sud ayant hérité de la légitimité, .... » ou encore STTS, A.18.128: «Lorsque les Jin s'établirent sur le fleuve Yangzi et la rivière Huai (c'est à dire au sud), ils étaient les détenteurs du calendrier (symbole de la légitimité). Les hordes qui les enviaient les appelèrent les brigands usurpateurs... ». 
Wei Shou - lorsqu'il aborde les périodes de fragmentation territoriale, en commençant par l'Antiquité pré-impériale :

Dans l'Antiquité, les feudataires se faisaient la guerre ; les victoires et les défaites étaient imprévisibles. [Les historiographes] notaient les réussites des autres États et ne cachaient pas les échecs des leurs. [En revanche,] depuis un proche passé, une telle équité n'est plus de mise. Les États proclament leurs propres triomphes et dénigrent les autres pays. Parce que la maison des Yuan 元 était issue des confins barbares et qu'elle avait été outragée par les Chinois, Wei Shou l'a élevée au dessus des autres et l'a comparée aux Zhou et aux Han. [Quant aux États établis à] Jianye 建鄴, il les a injustement dévalorisés, les comparant à des barbares du Sud. Lorsque des pays ennemis se font la guerre, les fers se croisent et les rancœurs se nouent. On peut calomnier à coup de lettres et de décrets, mais dans les textes [historiques], on ne doit pas déraisonner. Comment parler d'histoire à ceux qui n'ont pas compris ce point ? ${ }^{48}$

Liu Zhiji réitère la critique à plusieurs reprises, variant la forme, mais conservant le fond : à ses yeux Wei Shou est coupable d'avoir, par son histoire, légitimé un État qui ne devait pas l'être, un État barbare de surcroît :

Si on corrige les œuvres composées récemment - comme celles de Wang Shen 王沈, Sun Sheng 孫盛, Boqi 伯起 (Wei Shou) ou [Linghu] Defen 德㯣 - à la lumière des indications des sages du passé, [on s'aperçoit] qu'en matière de légitimité impériale, elles prennent le parti des rebelles et calomnient les loyalistes, que, dans leur description des États, elles rabaissent les pouvoirs légitimes et exaltent l'usurpation, et que pour les modes de vie, elles vantent les mœurs barbares au détriment des coutumes chinoises. Et ce ne sont là que les grandes lignes. Si l'on devait examiner les choses de manière approfondie et relever toutes les fautes, on verrait qu'elles sont aussi nombreuses que les cheveux sur notre tête : la tâche serait impossible ! ${ }^{49}$

Comme je l'ai souligné, puisque Wei Shou considère les Wei comme légitimes, les pouvoirs du Sud ne peuvent apparaître, dans ses écrits, que comme des nations illégitimes, voire vassales. Et 1'historien va très loin dans son entreprise de délégitimation : il les attaque à leur fondement, qui

${ }^{48}$ STTS, A.25.184.

${ }^{49}$ STTS, A.29.213. 
est, pour lui, la prise de la dignité impériale par l'empereur Yuan des Jin (Sima Rui 司馬頻, r. 317-323), fondateur des Jin dits orientaux ${ }^{50}$. Sapant cette base en présentant ce souverain comme un bâtard, Wei Shou laisse entendre que ces Jin ne sont pas les successeurs légitimes des Jin occidentaux (ceux installés à Luoyang). Par ricochet, les dynasties du Sud postérieures (Song, Qi et Liang) se trouvent aussi délégitimées, puisque l'un des maillons de la chaîne des abdications rituelles (shanrang 禪讓) - le mode normal de changement dynastique à cette époque - a été sectionné. Voici le début de la biographie de Sima Rui dans le Livre des Wei :

Sima Rui, des Jin usurpés, a pour prénom social Jingwen 景文. Il est le fils de Niu Jin 牛金, un général des Jin. Dans un premier temps, l'empereur Xuan 宣 des Jin engendra [Sima] Zhou 伷, grand général et prince $\mathrm{Wu}$ de Langya ; Zhou engendra [Sima] Jin 覲, Vice président surnuméraire et Prince Gong de Langya. L'épouse de Jin était une Xiahou 夏侯 de la principauté de Qiao 譙 et avait pour prénom social Tonghuan 銅環. Elle eut des rapports illicites avec [Niu] Jin et enfanta Rui, qui prit le patronyme de Sima et fut [présenté comme] le fils de [Sima] Jin. C'est de là que Rui prétend être originaire de Wen 溫 dans le Henei 河內. ${ }^{51}$

Cette histoire de bâtardise est insupportable pour Liu Zhiji. Ce passage lui donne l'occasion d'attaquer Wei Shou sur sa détestation des États du Sud : la haine de l'historien le pousserait à exploiter tout ce qui pourrait leur être défavorable, même s'il s'agit d'affabulations :

Récemment, Shen Yue 沈約, dans son Livre des Jin, s'est plu à créer de toutes pièces des théories saugrenues. Quand il a affirmé que l'empereur Yuan était le fils de Niu Jin, c'était pour se conformer à une prophétie qui stipulait que « le buffle (niu) succédera au cheval $(m a) »$ 牛繼馬後 ${ }^{52}$. [...] Wei Shou, qui détestait profondément les États du Sud, prenait plaisir à en relater les mauvais côtés. Aussi, lorsqu'il rédigea la «biographie de Sima Rui », reprit-il intégralement ce qu'on avait dit [sur la paternité de

${ }^{50}$ Sur la transition des Jin occidentaux aux Jin orientaux, $c f$. l'article de François Martin dans le présent volume.

${ }^{51}$ Weishu, juan 96, p. 2091. Wen dans le Henei est le lieu d'origine du clan Sima.

${ }^{52}$ Le Livre des Jin (Jinshu) de Shen Yue resta inachevé et ne nous a pas été transmis. L'auteur mentionne toutefois cette tradition sur la bâtardise de Sima Rui dans Songshu 宋書, Beijing : Zhonghua shuju, 1974, juan 27, p. 783. 


\section{Damien Chaussende}

Niu Jin]. [...] De telles absurdités sont extrêmement nombreuses. Si nous n'avions aucun élément [pour les récuser], nous serions bien en peine de distinguer le vrai du faux. ${ }^{53}$

La même critique apparait dans le chapitre A.15, « La collecte des matériaux » (caizhuan 採撰), suivie des lignes suivantes, particulièrement hargneuses : « On constate que [Wei Shou] n'eut pas de descendance et que ses ossements furent profanés après sa mort. C'est certainement la sanction des forfaits qu'il commit $\gg{ }^{54}$.

Wei Shou ne s'en prend pas qu'à Sima Rui ; il accorde beaucoup de place dans son œuvre aux autres souverains du Sud, auxquels il consacre de nombreuses biographies. Il est par ailleurs très explicite dans les intitulés de ces parties : si le fondateur des Jin orientaux est « Sima Rui, des

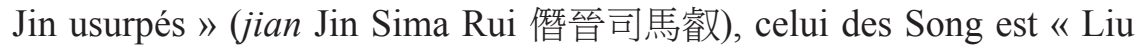
Yu, barbare des îles » (daoyi Liu Yu 島夷劉裕), de même pour Xiao Daocheng 蕭道成, fondateur des Qi du Sud et Xiao Yan 蕭衍, fondateur des Liang ${ }^{55}$. Les souverains d'autres États de l'époque ont également droit à des intitulés circonstanciés. Certains sont relativement neutres, comme ceux des chefs non-chinois, dont les noms sont précédés de simples ethnonymes : « Li Xiong le Cong 》 cong Li Xiong 寊李雄, « Tufa Wugu le Xianbei 》xianbei Tufa Wugu 鮮卑秃髮烏孤 ou encore « Juqu Mengxun le lushuihu» 盧水胡沮渠蒙遜. Les chefs chinois, quant à eux, reçoivent des qualificatifs peu flatteurs : si Huan Xuan 桓玄, qui manqua de peu de prendre le pouvoir à la fin des Jin, est aussi un « barbare des îles », Feng $\mathrm{Ba}$ 馮跋 (le fondateur des Yan du Nord) est quant à lui un «barbare des mers 》 (haiyi 海夷), et Zhang Shi 張定 un «soi-disant gouverneur de la province de Liangzhou » (sishu Liangzhou mu Zhang Shi 私署涼州牧張 定). De même pour Li Hao 李畗.

Ces intitulés sont non seulement une innovation de Wei Shou, mais ils sont révélateurs de l'insistance avec laquelle ce dernier souhaite discréditer les États dirigés par ces chefs. En refusant de désigner les souverains Sima (famille impériale des Jin), Liu (Song) et Xiao (Qi puis Liang) par leurs titres posthumes impériaux, il les rabaisse au rang de sujet. Liu

\footnotetext{
${ }^{53}$ STTS, B.8.458-459.

${ }^{54}$ STTS, A.15.107.

${ }^{55}$ Weishu, juan 97, p. 2129, juan 98, p. 2161 et 2171.
} 
Zhiji commente à plusieurs reprises ce procédé, qu'il trouve contraire à la vérité, et qui par ailleurs surcharge inutilement le texte. Dans le chapitre A.11, « Les intitulés » (timu 題目), après avoir critiqué Fan Ye, auteur du Livre des Han postérieurs, pour ses intitulés de chapitres trop développés, voici ce qu'il dit de Wei Shou :

Wei Shou suivit cette voie et alla même encore plus loin. Dans son histoire des Wei, chaque fois qu'il évoque un personnage originaire d'un État voisin, il mentionne après le nom, le pays et la fonction. Ainsi, pour les empereurs des dynasties du Sud, il écrit par exemple : « Sima Rui, des Jin usurpés » ou encore « Liu Yu, barbare des îles ». [...] De telles remarques figurent non seulement à l'intérieur des chapitres, mais également en tête de chacun d'eux. Agir ainsi, ce serait comme si, dans les deux histoires des Han (le Livre des Han et le Livre des Han postérieurs) ou dans la Monographie des Trois Royaumes, on aurait intitulé les biographies consacrées aux brigands : «Xiang Yu, hégémon usurpateur du royaume occidental de Chu » 西楚霸王項羽 ou 《Wei Xiao, roi usurpateur de Ningshuo » 偽 寧朔王隗㗊 [...] Les Anciens s'inquiétaient de la surabondance des lois. N'a-t-on pas atteint le comble de la profusion avec les intitulés de Fan [Ye] et de Wei [Shou] ? Si un auteur prise autant les détails au détriment des grandes lignes, il nous sera bien difficile de lui parler du style allusif [des Printemps et Automnes], où l'on parvient, en un mot, à exprimer le blâme ou la louange. ${ }^{56}$

Au-delà des intitulés, les exagérations de Wei Shou vont plus loin encore dans le corps des biographies, où il dévalorise considérablement les territoires sur lesquels règnent les souverains du Sud, en reprenant à l'envi l'ensemble des topoi dépréciatifs sur cette région. La biographie de Sima Rui est édifiante à cet égard. Wei Shou y relate les conditions dans lesquelles ce souverain constitua une cour impériale à Nankin en 317. Pour Wei Shou, cette cour n'était qu'une pâle réplique de celle du Nord, entendons, celle du Dai 代, le pouvoir politique à l'origine des Wei.

$\mathrm{Au}$ début du règne de l'empereur Pingwen 平文 ${ }^{57}$, Rui prit le titre de prince de Jin 晉王, proclama l'ère Jianwu, établit un Temple des

${ }^{56}$ STTS, A.11.86-87.

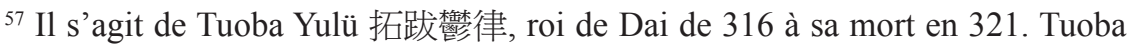
Yulü reçut le titre posthume d'empereur Pingwen des Wei en 399, lorsque Tuoba Gui, son descendant, accéda à la dignité impériale et fonda formellement la dynastie impériale des Wei. 


\section{Damien Chaussende}

ancêtres impériaux et des autels du sol et des moissons. Il créa une administration et nomma son fils Shao 紹 comme héritier présomptif. En tant que prince de Jin, il célébra le sacrifice jiao au sud. Cette même année, il usurpa la dignité impériale et ouvrit l'ère Daxing. Les cérémonies de la cour et les institutions de la capitale étaient imitées de ceux des souverains [légitimes] et calquées sur les pays du centre 中國 (c'est à dire ceux du Dai).

Poursuivant dans cet esprit, Wei Shou déprécie la région dont Sima Rui est le maître, le Sud, un territoire quasi invivable et à peine civilisé. Pour ce faire, il retrace en quelques lignes son histoire telle qu'elle apparaît dans les Classiques, en insistant tout particulièrement sur les données négatives. Il rappelle que ce Sud lointain était une terre étrangère à la civilisation chinoise et le lieu de toutes les usurpations.

[Rui] établit sa capitale à Danyang 丹陽, ancien territoire de Sun Quan 孫權 correspondant au Yangzhou 揚州 des tributs de Yu 禹 et éloigné de Luoyang de deux mille sept cents $l i$. Les montagnes et les cours d'eau y abondent ; les oies sauvages y nichent en nombre ; la terre n'y est que boue humide ; les champs de piètre qualité. [C'est le pays] des vêtements de toile venus des îles, comme il est dit [dans le Classique des documents]. Sous les Printemps et automnes, ce lieu correspondait à $\mathrm{Wu}$ 吳 et à Yue 越, deux pays dont les souverains usurpèrent le titre de Roi. Relégués dans un coin [du monde, ces royaumes] n'avaient jamais entendu parler des terres chinoises. [...] Ce territoire est immense et s'étend sur plusieurs milliers de li. Rui n'a fait que se concilier les populations qui y vivaient, rien de plus. Il n'est jamais parvenu à les avoir sous sa domination. Ce pays possède des rizières, mais les plantations sur sol sec y sont rares. On y vit de la pêche. Les gens sont rusés et profiteurs ; rares sont ceux qui font montre de bienveillance désintéressée. On ne fait pas de réserves de vivres, aussi y souffre-t-on souvent de la faim. Le climat y est chaud et humide si bien qu'œdèmes et diarrhées sont courants. L'air y est vicié par des miasmes pestilentiels ; le pays connaît toutes les formes d'attaques d'insectes et de bestioles.

Wei Shou, dans son opposition nord-sud, s'inscrit dans un contexte littéraire et historiographique dont il n'est pas, tant s'en faut, l'unique représentant ${ }^{58}$. Les sources de l'époque contiennent de nombreux passages

${ }^{58}$ Sur cette question, $c f$. la communication de François Martin, « Poisson ou mouton? Thé ou yaourt? Note sur quelques attitudes mentales à l'époque de 
révélateurs des points de vue des gens du Nord sur ceux du Sud et inversement. Yang Xuanzhi 楊衒之 ( $f$ l. 550), dans son Mémoire sur les monastères bouddhiques de Luoyang (Luoyang qielanji 洛陽伽藍記), nous rapporte un échange particulièrement intéressant à cet égard. L'affaire a lieu à Luoyang en 529 ; les débatteurs sont Chen Qingzhi 陳慶之, un ambassadeur envoyé par l'empereur Wu des Liang, et Yang Yuanshen 楊元 慎, un Chinois de la plaine centrale fonctionnaire des Wei du Nord. Après que Chen Qingzhi eut affirmé la supériorité des Liang, seule dynastie légitime à ses yeux, Yuanshen prend la parole :

Le Sud, provisoirement tranquille, est retiré dans un coin. C'est un pays très humide, grouillant de vermine, une région de miasmes et d'épidémies. La grenouille et la tortue habitent le même trou ; les hommes et les oiseaux y vivent en bandes. Les chefs ont les cheveux courts et n'ont l'air de rien; les indigènes sont tatoués et leur constitution est misérable. Naviguer sur les trois rivières, godiller sur les cinq lacs, n'est pas bénéficier de la civilisation ; aucune institution ne saurait les réformer. Bien que les descendants des relégués des Qin 秦 et des bagnards des Han 漢 y mêlent des mots de la plaine centrale, le baragouin de Min 閩 et de Chu 楚 n'est pas corrigé pour autant ! On a beau instituer des chefs et des sujets, les supérieurs sont des fainéants et leurs subordonnés des brutes ! [...] Nous, les Wei, nous avons reçu les registres et les chartes du ciel, nous avons fondé (un État) dans la région du mont Song 嵩 et de la rivière Luo 洛; les cinq pics en sont les pacificateurs et les quatre mers la famille. Nos règlements pour réformer les mœurs et amender les usages valent ceux des cinq empereurs. L'excellence de nos rites et de notre législation surpasse celle des cent princes. Vous, les compagnons des poissons et des tortues, comment pourriez-vous vénérer la justice et vous rendre à la cour, boire l'eau de nos étangs et manger de notre grain ? Quelle insolence de venir jusqu'ici ! ${ }^{59}$

Voyons un autre exemple, tiré du Livre des Qi du Sud, qui expose le point de vue d'un aristocrate du Sud sur les Wei. Sous le règne de l'empe-

la partition nord-sud », présentée lors du colloque " Pratiques culturelles et vie sociale sous les Six Dynasties » (INALCO/UMR 8583, 24 et 25 juin 2004). La communication est à paraître dans les actes du colloque.

${ }^{59}$ Luoyang qielan ji jiaoshi 洛陽伽藍記校釋, édition de Zhou Zumo 周祖謨, Beijing : Zhonghua shuju, 1963, juan 2, p. 105-107. Traduction de Jean-Marie Lourme, Mémoire sur les monastères bouddhiques de Luoyang, Paris : Les Belles Lettres, à paraître, chapitre XXII, p. 9. 
reur Wu 武 (r. 482-493), les Wei du Nord - c'est alors l'époque de l'empereur Xiaowen 孝文 (r. 471-499), le grand promoteur de la culture chinoise - demandent à Qi des livres, y compris les Classiques et autres ouvrages fondateurs ${ }^{60}$. La cour conseille au souverain de ne pas agréer la demande, mais Wang Rong 王融, un jeune lettré particulièrement talentueux, envoie un mémoire au trône et soutient qu'au contraire, il faut envoyer des livres aux Wei. On y trouve tout d'abord une présentation des Tuoba, riche de métaphores animales :

Les ennemis [Wei] ont des visages d'hommes, mais des cœurs de bêtes. Brutaux tels des loups, vénéneux tel des guêpes, ils s'opposent à l'ordre céleste et désobéissent aux règles terrestres. [...] Ils n'ont aucun penchant pour la sagesse et la bienveillance, ni pour les vertus d'effacement, de probité ou de cultivation de soi. Contrairement aux chiens et aux chevaux, ils sont indomptables; et leurs yeux brillent d'un regard de rapace ou de tigre.

Wang Rong explique ensuite qu'il serait judicieux de leur envoyer des livres, puisqu'ils les demandent eux-mêmes. Il avance deux arguments : premièrement, en leur donnant des livres, la civilisation chinoise pourra renaître dans ces territoires déculturés puisque passés aux mains des barbares :

Si nous les faisons venir à nous par la culture et la vertu et que nous leur offrons des copies de nos livres, les usages des Han renaîtront dans la capitale [du Nord] et sa couronne, ainsi les règles se transmettront dans la région métropolitaine puis traverseront la passe [de Hangu ${ }^{61}$. Et cela sans avoir eu besoin de lever nos huit cents armées et nos cent mille soldats et cavaliers. Pour sûr, les ennemis mettront alors leurs armes à notre service ; les Trois Qin ${ }^{62}$ seront reconquis et le pays réunifié.

L'autre argument est qu'au contact des livres, ces barbares se prendront les pieds dans les rites, pour ainsi dire, si bien qu'il sera plus aisé de les vaincre :

${ }^{60}$ Nan Qishu 南齊書, Beijing : Zhonghua shuju, 1972, juan 47, p. 818-820. Je remercie François Martin qui m'a indiqué ce passage sur Wang Rong.

${ }^{61}$ Passe qui marque la limite entre la plaine centrale, à l'est, et la vallée de la rivière Wei, à l'ouest.

${ }^{62}$ Une des désignations classiques du bassin de la Wei. 
Si on les vêt de robe de cour et de bonnets de cérémonie, qu'on les éduque en leur apprenant les échanges de politesse, qu'on leur apprend à avancer selon les rites, ils seront alors entravés comme dans une gangue, attentifs comme s'ils marchaient sur une glace mince au bord d'un gouffre, et tourneront en cercle comme s'ils dansaient. Ils seront tellement empêtrés qu'ils ne pourront plus avancer.

Des propos de ce type sont récurrents dans les sources du haut Moyen Âge ${ }^{63}$; l'une des spécificités du Livre des Wei par rapport aux histoires écrites dans le Sud est la place importante que Wei Shou accorde aux États méridionaux en terme de volume. Non seulement il en parle beaucoup, mais il remonte également loin dans le temps, puisqu'il commence à Sima Rui. Le Livre des Song et le Livre des Qi du Sud, composés respectivement sous les Qi et les Liang, contiennent bien chacun un chapitre consacré aux Wei, intitulé « Ennemis à la tête cordée » (Suolu 索虜) et «Ennemis Wei » (Weilu 魏虜) ${ }^{64}$, mais on n’y détaille pas les règnes des souverains à la manière de Wei Shou. On sent, chez ce dernier, une volonté de traiter longuement de ces États afin de mieux les détruire. Pour Liu Zhiji, Wei Shou commet par là un hors-sujet, d'autant plus risible que les Wei étaient, à l'origine, une simple chefferie : délégitimer les Jin orientaux n'a aucun sens. Dans le passage suivant, extrait du chapitre A.12, « La délimitation du sujet » (Duanxian 斷限), Liu Zhiji vise directement Wei Shou, sans le nommer :

Dès lors que les Barbares eurent promulgué leurs lois, le pays se fragmenta en plusieurs maisons [régnantes]. Les dynasties du Sud ayant hérité de la légitimité, elles exclurent les Wei et les autres Barbares. Aussi, [dans leurs écrits historiques], consacrèrent-elles de [simples] notices aux barbares Di

${ }^{63}$ Pour d'autres exemples, en particulier tirés des Instructions familiales pour le clan Yan (Yanshi jiaxun 顏氏家訓) de Yan Zhitui 顏之推 (ca. 531-591), un homme du Sud qui émigra au Nord, $c f$. "Sixth Century China", chapitre 2 de Arthur Wright, The Sui Dynasty, New York: Alfred A. Knopf, 1978, p. 32-36.

${ }^{64}$ Songshu, juan 95, p. 2321 ; Nan Qishu, juan 57, p. 983. Le Livre des Liang, rédigé au début des Tang, comporte des notices sur les États barbares (juan 54), mais aucune sur les Wei. La « tête cordée » est une allusion aux nattes que portaient les Tuoba, voir sur ce point la longue note dans Paul Demiéville, Le Concile de Lhasa : une controverse sur le quiétisme entre bouddhistes de l'Inde et de la Chine au vIII siècle de l'ère chrétienne, Paris : Collège de France, Institut des

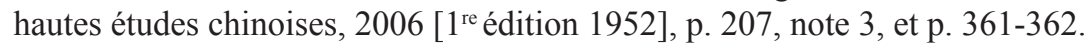




\section{Damien Chaussende}

氏 et Qiang 姜; les « Ennemis à la tête cordée » firent l'objet d'un chapitre ordinaire ${ }^{65}$. Les Wei, qui provenaient à l'origine d'une race indistincte, se prétendaient quant à eux les souverains légitimes. Leurs historiens firent corps avec leur dynastie et voulurent dépasser les œuvres de leurs prédécesseurs. Ils intégrèrent donc dans leurs écrits les Jin du Sud et les différentes cours illégitimes du Nord. Faisant d'eux de simples brigands, ils les confinèrent dans des chapitres ordinaires. Or, à l'époque des empereurs Yuan 元 et Ming 明 des Jin, lorsque les Qin 秦 et les Zhao 趙 occupaient la plaine centrale, le clan Yuan se prosternait et leur faisait allégeance, se déclarant leurs vassaux. Pourtant, ils les relèguent dans des chapitres ordinaires, c'est bien le comble de l'impudence ! ${ }^{66}$

D'un côté, Wei Shou sape la légitimité des pouvoirs du Sud en les attaquant à leur racine - le règne de Sima Rui - et en insistant sur le fait qu'ils apportent tribut à Wei ${ }^{67}$, et de l'autre, il fait son possible pour valoriser les Wei depuis leurs origines. Pour ce faire, il innove encore une fois par rapport aux autres histoires en consacrant un chapitre entier aux chefs Tuoba qui se succédèrent depuis les Jin occidentaux, voire depuis les Han postérieurs, soit bien avant la fondation des Wei en 399.

Liu Zhiji admet que, dans une histoire, on puisse remonter un peu avant la naissance de l'État traité, notamment lorsque certains ancêtres du fondateur ont joué un rôle important. C'est l'une des conséquences des nominations impériales posthumes, qui débutent avec les Cao-Wei : Cao Cao 曹操, n'ayant jamais régné de son vivant, fut nommé empereur $\mathrm{Wu}$ 武 de manière posthume par son fils Cao Pi 曹丕, l'empereur Wen 文, à la fondation des Wei. Par la suite, Chen Shou lui consacra des annales impériales au début de la Monographie des Trois Royaumes, confirmant par là son statut de père fondateur des Wei. De même, Sima Yi 司馬懿, Sima Shi 司馬師 et Sima Zhao 司馬昭, qui n'accédèrent jamais à la dignité impériale, furent faits empereurs par Sima Yan après leur mort, à la fondation des Jin, et eurent droit à des annales impériales dans le Livre des Jin. Pour Liu Zhiji,

${ }^{65}$ Le chapitre (ou biographie dans le cas d'un individu) ordinaire est le zhuan 傳, par opposition aux annales impériales ( $j i$ 紀).

${ }^{66}$ STTS, A.12.90.

${ }^{67}$ Par exemple dans Weishu, juan 4A, p. 71 : « Liu Yilong (empereur Wen des Song) envoya des émissaires porter le tribut. »; juan 7A, p. 151 : « Xiao Daocheng (empereur Gao des Qi) envoya des émissaires porter le tribut. » Liu Zhiji commente cette question dans STTS, A.22.166. 
ces exemples sont tout à fait justifiés :

Par ailleurs, certains [personnages] avaient le rang de simples sujets, mais leur œuvre égala celle d'un [véritable] souverain. Ainsi en alla-t-il de [...] Zhongda 仲達 (Sima Yi), de Shi 師 et de Zhao 昭 des Jin. Leur conférer des titres posthumes qui les hissaient au niveau des fils du Ciel était approprié. ${ }^{68}$

Tel n'est pas le cas des chefs tuoba :

Les Yuan, quant à eux, sortaient des confins nordiques ; leurs souverains n'étaient que des chefs de tribu. Mais lorsque l'empereur Daowu voulut honorer ses ancêtres, il [donna des titres posthumes impériaux] à vingthuit d'entre eux. Depuis les débuts de l'univers, cela ne s'était jamais encore fait. Les annales préliminaires du Livre des Wei respectent tous ces titres fallacieux. On y désigne ces chefs sous le titre d'empereur, et leurs décès sont notés avec le terme réservé aux fils du Ciel ${ }^{69}$. En quoi ces gens différaient-il de macaques qu'on aurait couronnés ou de rats putréfiés qu'on aurait fait passer pour du jade ? ${ }^{70}$

Liu Zhiji évoque ici le chapitre mentionné plus haut, qui ouvre le $\mathrm{Li}$ vre des $W e i^{71}$. Intitulé « Annales préliminaires » (Xuji 序紀), il consiste en une recension des souverains Tuoba qui furent nommés empereurs à titre posthume en janvier 399, lorsque Tuoba Gui 拓拔圭 (l'empereur Daowu) accéda à la dignité impériale et fonda formellement la dynastie des Wei ${ }^{72}$. De fait, les treize premiers souverains ne font l'objet que d'une simple mention, sans même de date. Le premier élément de datation concerne le quinzième souverain, Tuoba Liwei 拓拔力微 : Wei Shou rapporte qu'il aurait envoyé son fils en otage chez les Cao-Wei en 261.

$\mathrm{Au}$ fond, ce que reproche Liu Zhiji à Wei Shou n'est probablement

${ }^{68}$ STTS, A.14.100.

${ }^{69}$ Le terme spécifique désignant le décès d'un empereur est beng 崩.

${ }^{70}$ STTS, A.14.100-101.

${ }^{71}$ Weishu, juan 1, p. 18. Ce chapitre est intégralement traduit dans Jennifer Holmgren, Annals of Tai: Early T'o-pa History. An Annotated Translation of Chapter 1 of Wei Shu, Canberra: The Australian National University, 1982, ouvrage que je n'ai malheureusement pas pu consulter, aucune bibliothèque française ne le possédant.

${ }^{72}$ Weishu, juan 2, p. 34. 


\section{Damien Chaussende}

pas tant le fait d'avoir mentionné des souverains, que d'en avoir mentionné trop, et de les avoir excessivement valorisés en transmettant leur titre impérial posthume. Pour le critique, ces chefs tuoba, des « macaques couronnés », ne méritaient pas que Wei Shou s'y attarde. C'est plus largement la valorisation des coutumes tuoba que Liu Zhiji désapprouve, coutumes que Wei Shou compare ici et là à des précédents consignés dans les Classiques ou les ouvrages historiques anciens :

[Certains faits] ne sont pas aisés à déformer. Mais [des auteurs] inconvenants les polluent de leur encre et les rapportent en les déformant et en les maquillant, en particulier les historiens des époques récentes. Le Livre des Wei raconte, par exemple, que [1'empereur Daowu] monta sur le trône et donna des titres de fonctionnaire inspirés par des noms d'oiseaux. Ainsi est-il écrit que le souverain « estimant la sobriété, prit pour modèle antique Shaogao 少畽 " ${ }^{73}$. Lorsqu'il est question des unions matrimoniales entre les Wei et les peuples frontaliers, [le Livre des Wei] affirme que le monarque « souhaitant se gagner les cœurs des populations barbares, imita l'empereur Gao des Han 漢高 » ${ }^{74}$. On trouve de nombreux autres exemples de ce type. Les Wei étaient pourtant issus des confins nordiques, et ne connaissaient guère les classiques antiques. Aussi, lorsqu'ils s'allièrent aux barbares Man 蠻 et Yi 夷, ce n'était guère plus que les unions entre le Qin 秦 et le Jin 晉 ${ }^{75}$. Le fait d'avoir institué des fonctionnaires-oiseaux ne pourrait être comparé à ce dont parle Tanzi 邩子. ${ }^{76}$

Cette mise en valeur des Tuoba et de leur culture, cette insistance à enjoliver leur histoire est peut-être liée aux Gao eux-mêmes, et en particulier à Gao Huan. Les origines des Gao ne sont pas très claires. Gao Huan était peut-être un Xianbei sinisé. Il eut à cœur, en tout cas, lorsqu'il fut

${ }^{73}$ Un personnage légendaire mentionné dans le Zuozhuan, à l'année 525 av. J.-C., dont les ministres portaient des titres inspirés par des noms d'oiseaux. En reproduisant ce précédent, l'empereur Daowu se plaçait dans une tradition prestigieuse puisque mentionnée dans un classique.

${ }^{74}$ L'empereur Gao des Han, afin d'apaiser les relations entre l'Empire et ses voisins Xiongnu, maria nombre de princesses chinoises à des chefs xiongnu. L'empereur Daowu l'imita.

${ }^{75}$ Deux principautés des Printemps et Automnes dont les membres de l'aristocratie nouèrent de nombreuses unions matrimoniales.

${ }^{76}$ STTS, A.21.148. Tanzi, ce sage étranger apprécié de Confucius, avait évoqué les fonctionnaires-oiseaux dans un discours qu'il tint à $\mathrm{Lu}$. 
à la tête des Wei orientaux, de ménager l'antagonisme Chinois-Xianbei qui existait alors, n'hésitant pas à user d'un discours différent selon les populations ${ }^{77}$ :

Quand il s'adressait aux Xianbei 鮮卑, il leur disait : «Les Han 漢民 sont vos esclaves. Ils labourent pour vous. Leurs femmes tissent pour vous. Ils transportent pour vous grain et tissu. C'est grâce à eux que vous avez chaud et que vous êtes rassasiés. Comment pouvez-vous les traiter de haut comme vous le faites? ». Et quand il parlait aux Chinois 華人, il leur disait : « Les Xianbei sont vos hôtes. Pour une mesure de grain et une pièce de tissu que vous leur donnez, ils frappent vos ennemis pour vous, ils vous assurent la tranquillité. Comment pouvez-vous les haïr ainsi que vous le faites? $»^{78}$

L'épouse qui lui donna Gao Yang, l'impératrice Lou 婁 (501-562) était une Xianbei de pure souche. Le fondateur des Qi, celui qui commanda à Wei Shou son histoire, était donc un sang-mêlé 79 , et s'identifiait volontiers comme un xianbei ${ }^{80}$. La valorisation des Wei par Wei Shou est donc peut-être la résonance d'une vulgate de l'époque de Gao Huan, qui cherchait à se concilier les Xianbei (en particulier le clan impérial des Tuoba), et également une façon, directe celle-ci, de plaire à Gao Yang.

Quoi qu'il en soit sur ce point précis, et même si Liu Zhiji insiste surtout sur le parti pris pro-Nord de Wei Shou, les commentaires concernant son attachement aux Qi, nous l'avons vu, ne sont pas inexistants ; ils se rattachent à la question du traitement historiographique de la guerre civile des années 530 et de la bipartition subséquente des Wei.

${ }^{77} C f$. Étienne Balazs, «Études sur la société et l'économie de la Chine médiévale. I. Le traité économique du "Souei-Chou" ", T'oung Pao, 1953-42 (3/4), p. 252 et 255-259.

${ }^{78}$ Zizhi tongjian, juan 157, p. 4881 (année 537). Traduction inédite de François Martin.

${ }^{79} \mathrm{Ce}$ qui n'empêcha pas ce souverain, un fou sanguinaire à la Caligula, de faire exterminer, en 559, les membres encore vivants du clan Tuoba, soit 721 personnes. Cf. Étienne Balazs, opus cit., p. 259, note 2.

${ }^{80}$ Cf. Jennifer Holmgren, "Seeds of Madness : A Portrait of Kao Yang, First Emperor of Northern Ch'i, 530-560 A.D.", Papers on Far Eastern History, 1981-24, p. 120. 


\section{Damien Chaussende}

\section{Les Wei orientaux comme préhistoire des Qi}

Trois commentaires de Liu Zhiji, parfois répétés, portent de manière plus ou moins directe sur la façon dont Wei Shou composa son histoire dans une optique favorable aux Gao : la question de l'empereur réfugié auprès de Yuwen Tai, celle de la place accordée aux Gao dans le Livre des Wei et enfin le traitement du chef de guerre sanguinaire Erzhu Rong.

Rappelons qu'en 534, date qui marque la naissance des deux cours se réclamant des Wei, le pouvoir n'est plus dans les mains du clan impérial Tuoba. Les Wei orientaux sont dirigés par Gao Huan, qui règne à travers l'empereur fantoche Yuan Shanjian. Les Wei occidentaux sont quant à eux sous la tutelle de Yuwen Tai, qui a auprès de lui Yuan Xiu.

Or ce Yuan Xiu avait été placé sur le trône impérial par Gao Huan luimême en 532, après un série d'événements complexes dont voici la succession : d'abord, Gao Huan défit les Erzhu lors de la bataille de Hanling 韓陵, puis il déposa et tua Yuan Gong 元恭 (r. 531-532), mis sur le trône par Erzhu Shilong 爾朱世隆 en 531. Ensuite Gao Huan intronisa puis déposa presque aussitôt Yuan Lang 元朗 (r. 531-532). Enfin vint le tour de Yuan Xiu. Ce dernier quitta Gao Huan pour Chang'an car il ne supportait pas son rôle de marionnette ; il ne se doutait pas qu'il subirait le même sort chez Yuwen Tai, qui finit par le faire exécuter en 535, pour le remplacer par Yuan Baoju 元寶 炬 (r. 535-551), un autre pantin du clan impérial des Wei.

Cette série d'empereurs, d'anti-empereurs ou de souverains déposés donna du fil à retordre, pour ainsi dire, aux historiographes postérieurs chargés de distinguer les véritables monarques légitimes des usurpateurs. Dans le Livre des Wei, Yuan Gong et Yuan Lang ont droit à des annales impériales (ce qui fait d'eux des empereurs légitimes aux yeux de l'historien) ; il ne sont toutefois pas désignés par des noms posthumes, mais par l'appellation d'« empereurs déposés » (respectivement premier et second empereur déposé [qianfeidi 前廢帝 et houfeidi 後廢帝] ${ }^{81}$ ), ce qui correspond à la réalité des faits. Yuan Baoju est totalement délégitimé : il est désigné, dans le corps de l'ouvrage, par son prénom personnel et éventuellement son titre princier (le prince de Nanyang 南陽王) et n'a droit à aucune biographie propre. Dans le Livre des Wei tel qu'il nous a été transmis, les annales de Yuan Shanjian (où il est désigné par son nom posthume d'empereur Xiaojing 孝

${ }^{81}$ Weishu, juan 11, p. 273 et 278. 
靜帝) appartiennent aux chapitres restitués sous les Song ${ }^{82}$; elles ne sont pas de la main de Wei Shou. Cependant, dans bien d'autres endroits de son ouvrage, celui-ci utilise cette appellation, notamment dans des dates ${ }^{83}$. Il est certain que dans les annales originales, 1'historien désignait ce souverain par ce titre posthume.

Jusque là, il s'agit de cas relativement clairs du point de vue politique : ces souverains étaient soit légitimes, soit ne l'étaient pas. Le cas de Yuan Xiu est plus complexe : mis sur le trône par Gao Huan, Wei Shou ne peut le délégitimer totalement (ce serait accuser Gao Huan d'avoir intronisé un usurpateur), et doit donc lui accorder des annales impériales ${ }^{84}$. Mais ce souverain est allé se réfugier dans une cour qui, elle, est illégitime. Aussi, Wei Shou trouve-t-il une solution intermédiaire : il désigne Yuan Xiu comme «l'empereur fuyard ${ }^{85}$ (chudi 出帝), une première dans le corpus des histoires officielles, faisant de ce souverain un monarque à demi légitime. Liu Zhiji commente cette innovation dans le chapitre A.14, « Les appellations » (chengwei 稱謂) :

Wei Shou fut le seul à ne suivre ni les modèles des Anciens, ni les usages récents [dans ses appellations]. Agissant selon son gré, il ne s'imposa aucune règle. Dans son Livre des Wei, il désigne le prince de Pingyang 平陽 (Yuan Xiu) comme « l'empereur fuyard» et les Sima par l'expression « les usurpateurs Jin ». Il appelle « Barbares des îles » les [souverains du Sud] à partir de Huan [Xuan] et des Liu. Pour flatter les Qi, il rabaisse [la cour installée] à l'ouest des passes. Prenant parti pour les Wei du Nord, il calomnie tant qu'il peut les dynasties du Sud. Ses haines et ses sympathies transparaissent dans les moindres détails [de son œuvre]. Il distribue louange et blâme de manière arbitraire; ses propos n'ont aucun fondement. Les appellations qu'il emploie ont pour seul but d'impressionner. [...] Quand une chose est déraisonnable, il est difficile d'en imposer la pratique. Si, comme Wei Shou, on propose des appellations trompeuses qui ne reposent sur aucun précédent, fussent-elles publiées dans des livres, elles ne sauraient se transmettre. ${ }^{86}$

${ }^{82} C f$. la note des éditeurs de la Zhonghua shuju, Weishu, juan 12, p. 315, note 1.

${ }^{83}$ Par exemple (il y en a beaucoup), Weishu, juan 14, p. 352 ; juan 19, p. 488 ; juan 68, p. 1518.

${ }^{84}$ Ces annales se trouvent dans Weishu, juan 11, p. 281-292.

${ }^{85}$ Weishu, juan 11, p. 281.

${ }^{86}$ STTS, A.14.101. 


\section{Damien Chaussende}

L'Histoire des dynasties du Nord offre un contraste intéressant du point de vue des intitulés des annales impériales et des appellations des souverains, fort différents de ceux choisis par Wei Shou. C'est que son auteur, Li Yanshou, regrettait que les différentes histoires des États du haut Moyen Âge soient toutes si orientées idéologiquement. Il reprenait en cela certaines des idées de son père, Li Dashi 李大師, qu'il évoque dans la postface (xuzhuan 序傳) de l'ouvrage :

Dashi dès sa jeunesse avait l'ambition d'écrire. Il considérait que, sous les Song, les Liang, les Chen, les Wei, les Qi, les Zhou et les Sui, pendant la division Nord-Sud, les [populations du] Nord étaient désignées par le terme « ennemis à la tête cordée » dans les ouvrages du Sud et les méridionaux étaient appelés « barbares des îles » dans les livres du Nord. [Dans ces ouvrages], on traitait son État avec moult détails ; les notations sur les autres pays étaient en revanche approximatives, voire inexactes. [Dashi] souhaitait corriger [ces écrits] et composer des annales sur le modèle des Printemps et automnes de Wu et Yue 吳越春秋 qui traiteraient de manière approfondie du Nord et du Sud. ${ }^{87}$

Li Yanshou s'efforça d'écrire deux histoires plus neutres que celles composées avant lui. Dans l'Histoire des dynasties du Nord, tous les souverains que j'ai mentionnés plus haut sont désignés par leur nom posthume, dans la mesure du possible : Yuan Gong y est l'empereur Jiemin 節閔 帝, Yuan Xiu, l'empereur Xiaowu 孝武帝, Yuan Baoju, l'empereur Wen des Wei occidentaux 西魏文帝 et Yuan Shanjian, l'empereur Xiaojing des Wei orientaux 東魏孝靜帝 ${ }^{88}$. Seul Yuan Lang continue à être désigné par la formulation d'empereur déposé, sans doute parce qu'il ne reçut jamais de véritable nom posthume.

Les intitulés et les appellations sont le symptôme le plus manifeste de la légitimité accordée aux souverains par les historiens. Le Livre des Wei ne possède pas de biographies, ou encore moins d'annales, aux membres du clan Gao ayant permis la fondation des Qi, en particulier Gao Huan : celui-ci n'est encore officiellement, sous les Wei, qu'un ministre, un général et un régent, mais il est tout de même très présent dans l'ouvrage.

Liu Zhiji affirme à deux reprises (dans la notice générale, puis dans

${ }^{87}$ Beishi, juan 100, p. 3343.

${ }^{88}$ Voir la table des matières du juan 5 du Beishi. 
la citation ci-dessus) que Wei Shou «flatte les Qi » (chan Qi 諂齊). C'est une manière de dire qu'il écrit ce que les Gao (Gao Yang en l'occurrence) veulent entendre, d'une part, sur les Wei, et d'autre part sur eux-mêmes. Liu Zhiji est un peu plus explicite dans ce passage tiré du chapitre A.12, « La délimitation du sujet » :

Dans une histoire consacrée à une seule dynastie, il arrive que des événements antérieurs viennent s'entrelacer avec ceux relatés. Lorsqu'ils ont déjà été exposés dans un autre écrit, il n'est nul besoin de les répéter. C'est pourquoi la soumission de Ziying 子嬰 à Pei 沛 (Gaozu des Han ${ }^{89}$ ) n'est détaillée que dans les annales des Qin (des Mémoires historiques de Sima Qian) et que la mort de Bofu 伯符 (Sun Ce), à la fin des Han ${ }^{90}$, est traitée uniquement dans le 《Livre de $\mathrm{Wu}$ » 吳書 (de la Monographie des Trois Royaumes de Chen Shou). [Or] lorsque Shen [Yue] 沈 traite des Jin, il remonte jusqu'aux souverains Liu 劉 ${ }^{91}$; quand Wei Shou écrit sur les Wei du Nord, il va jusqu'à prendre en compte les princes Gao. Or, le royaume de Shu 蜀 et les Qi 齊 ont chacun leur histoire. Outrepasser ainsi les limites d'une histoire pour les y faire figurer, est-ce bien une pratique convenable? ${ }^{92}$

Ces lignes, bien qu'elle mentionnent nettement les princes Gao, ne permettent pas de bien comprendre à quoi fait allusion précisément Liu Zhiji. Pour Pu Qilong 浦起龍 (1679-1762), l'auteur du Shitong tongshi 史 通通釋 (Explication complète du Traité de l'historien parfait), il s'agirait de références à Gao Huan. Ce dernier étant le régent de Yuan Shanjian

${ }^{89}$ Ziying était le fils du second empereur des Qin. Il régna un peu plus d'un mois, puis se déclara vassal du futur empereur Gaozu des Han. Il fut finalement tué par l'adversaire de ce dernier, Xiang Yu.

${ }^{90}$ Sun Ce mourut en l'an 200, donc en tant que sujet des Han. Sa biographie figure cependant dans la Monographie des Trois Royaumes, de Chen Shou, et non dans le Livre des Han postérieurs, qui fut rédigé un siècle et demi plus tard, au milieu du ve s.) de Fan Ye.

${ }^{91}$ Shen Yue composa un Livre des Jin qui ne nous a pas été transmis. D'après ce que dit Liu Zhiji, il aurait fait commencer son ouvrage non à la fondation de cette dynastie en 266, mais un peu plus tôt, en intégrant la conquête du royaume de Shu (aux mains de la famille Liu) par les Wei.

${ }^{92}$ STTS, A.12.89-90. 
jusqu'à sa mort en 547 , les annales de ce souverain ${ }^{93}$ correspondent à la période de régence. Elles sont effectivement centrées sur la figure de Gao Huan et consiste moins en des annales de l'empereur des Wei, qu'en une relation des hauts faits de son régent. Ce chapitre n'est malheureusement pas de la main de Wei Shou, il est l'un de ceux restitués sous les Song ${ }^{94}$; mais l'on peut raisonnablement penser qu'étant donné l'orientation générale de l'ouvrage de Wei Shou, le texte d'origine devait probablement mettre également en avant Gao Huan.

D'autres passages, non restitués sous les Song, sont également clairement orientés en faveur de Gao Huan ; on s'en aperçoit en comparant le Livre des Wei avec l'Histoire des dynasties du Nord. Nombre de ces divergences ont été mises en lumière par le grand philologue Zhao Yi 趙 翼 (1727-1814) dans ses Notes sur les Vingt-deux histoires (Nian'er shi zhaji 廿二史答記) ${ }^{95}$. J'en donnerai ici quelques exemples, à titre d'illustration. Selon Zhao Yi, Wei Shou livre un récit pro-Gao lorsqu'il célèbre une victoire de Gao Huan contre Xing Gao 邢杲 ${ }^{96}$, un élément absent de 1'Histoire des dynasties du Nord. Lors du massacre de Heyin en 528, Wei Shou affirme que Erzhu Rong souhaitait usurper la dignité impériale ; il en fut dissuadé par Gao Huan et Sima Ziru 司馬子如 ${ }^{97}$. Or, 1'Histoire des dynasties $d u$ Nord n'évoque pas du tout Gao Huan dans l'affaire, et attribue tous les mérites à Liu Lingzhu 劉靈助 ${ }^{98}$. Là encore, le texte de Wei Shou met en valeur Gao Huan, qui apparaît comme un défenseur des Wei.

Le choix des mots peut-être aussi particulièrement révélateur. Par exemple, en 531, Gao Huan se retourne contre les Erzhu, dont il avait été l'allié jusque là. Wei Shou, dans les annales de Yuan Gong (le premier empereur déposé), écrit :

${ }^{93}$ Weishu, juan 12, p. 297-314.

${ }^{94} \mathrm{Cf}$. la note des éditeurs de la Zhonghua shuju, Weishu, juan 12, p. 315, note 1.

${ }^{95}$ Cf. le chapitre 168 《Les nombreuses falsifications du Livre des Wei 》(魏書 多曲筆) dans Nian'er shi zhaji jiaozheng 廿二史箚記校證 (Notes sur les vingtdeux histoires), édition de Wang Shumin 王樹民, Beijing : Zhonghua shuju, 1984, juan 13, p. 263-264.

${ }^{96}$ Weishu, juan 10, p. 261.

${ }^{97}$ Weishu, juan 74, p. 1648.

${ }^{98}$ Beishi, juan 48, p. 1754. 
Le prince Xianwu de Qi (Gao Huan), parce que les Erzhu s'étaient rebellés, leva à Xindu, pour la première fois, les armées de la justice. 齊獻武王 以尔朱逆亂，始興義兵於信都. 99

« Lever les armées de la justice » est une expression particulièrement laudative : Gao Huan est ici dans le camp du bien. Li Yanshou, dans le passage correspondant dans l'Histoire des dynasties du Nord, raconte les choses différemment, et ne cherche pas à mettre en valeur Gao Huan :

Gao Huan, prince de Bohai, leva ses troupes à Xindu, affirmant qu'il le faisait pour châtier les Erzhu. 勃海王高歡起兵信都, 以誅尔朱氏為名. ${ }^{100}$

Les procédés utilisés par Wei Shou pour mettre en valeur ou couvrir les Gao ne lui sont pas spécifiques, et sont le lot de nombreux auteurs d'histoires officielles. Par exemple, l'auteur de la Monographie de Trois Royaumes, Chen Shou, usa du même type d'expédients dans son traitement des Sima, le clan fondateur des Jin, sous le règne desquels il écrivit son œuvre. Il ne pouvait bien entendu pas en dire du mal, et devait aussi, d'une façon ou d'une autre, les mettre en valeur ${ }^{101}$.

Les histoires du Sud ne sont pas en reste non plus. Je citerai deux exemples tirés du Livre des Liang ${ }^{102}$. Yao Silian 姚思廉 (557-637), son auteur, cherche à ne pas nuire à 1'empereur Yuan 元 des Liang (Xiao Yi 蕭繹, $r$. 552-555), un souverain particulièrement contesté dans l'Histoire des dynasties $d u$ Sud. La raison en est que son père, Yao Cha 姚察 (533606), fut très attaché aux Liang, et ce fut lui qui commença la rédaction du Livre des Liang. Aussi l'ouvrage est-il particulièrement orienté en faveur de la dynastie et de ses souverains, ce qui n'est pas le cas de l'Histoire des dynasties du Sud. Dans ce dernier ouvrage, les annales consacrée à Xiao Yi rapportent les circonstances de sa conception de manière peu propre à lui faire honneur : son père, l'empereur Wu des Liang, se serait rué sur sa mère, une simple femme du palais, après qu'une brise eut soulevé son

${ }^{99}$ Weishu, juan 11, p. 277.

${ }^{100}$ Beishi, juan 5, p. 168.

${ }^{101}$ Cf. Damien Chaussende, Des Trois Royaumes aux Jin, p. 62-68, 201, 210215.

102 Je remercie François Martin de m'avoir indiqué ces passages. 


\section{Damien Chaussende}

vêtement ${ }^{103}$. Autre exemple, la biographie de Wang Wei 王偉 laisse entrevoir la cruauté extrême du monarque : après un accès de colère, il fit clouer Wei par la langue à un pilier, lui fit ouvrir le ventre et le fit dépecer vivant ${ }^{104}$. Les parties correspondantes dans le Livre des Liang ne rapportent pas de telles informations sur l'empereur ${ }^{105}$.

Dans le Livre des Wei, Wei Shou couvre non seulement les Gao, mais également Erzhu Rong, que Gao Huan avait suivi, pour ensuite après la mort de Rong - se retourner contre son clan. Erzhu Rong s'est rendu célèbre dans la littérature de cette époque parce qu'en 528, il avait pris le pouvoir des mains de l'impératrice douairière Ling 靈太后, née $\mathrm{Hu}$, et fait massacrer l'ensemble de la cour, qu'il avait réunie près de Luoyang, à Heyin. Il fit noyer l'impératrice ainsi que l'empereur fantoche (un tout jeune enfant) qu'elle avait mis sur le trône pour évincer Yuan $\mathrm{Xu}$ 元詡 (emp. Xiaoming 孝明, $r$. 515-528) ${ }^{106}$. La mort de Rong en 530, tué par le monarque qu'il avait mis sur le trône, Yuan Ziyou 元子敒 (emp. 孝莊, $r$. 528-531), laissa les coudées franches à Gao Huan, qui combattit le clan de Rong en prétendant défendre la maison de Wei.

Le traitement d'Erzhu Rong par Wei Shou est pour le moins étonnant au premier abord : la biographie est clairement orientée en faveur de l'homme, et le jugement que l'on trouve à la fin, extrêmement flatteur ${ }^{107}$. Liu Zhiji mentionne cette question à deux reprises. La première fois dans le chapitre A.9, «Les jugements récapitulatifs » ${ }^{108}$ (lunzan 論贊) :

Certaines évaluations sont hors de propos et manquent de justesse.

${ }^{103}$ Nanshi 南史, Beijing : Zhonghua shuju, 1975, juan 8, p. 234.

${ }^{104}$ Nanshi, juan 80, p. 2018-2019.

${ }^{105}$ Liangshu 梁書, Beijing : Zhonghua shuju, 1973, juan 5, p. 138-136, et juan 56, p. 864 pour le passage sur Wang Wei.

${ }^{106}$ Outre le Weishu, un récit des événements se trouve dans le Mémoire sur les monastères bouddhiques de Luoyang, trad. J.-M. Lourme, II.24-33 ; Luoyang qielanji, juan 1, p. 29-35.

107 Weishu, juan 74, p. 1656-1657.

${ }^{108}$ Les jugements récapitulatifs sont de courtes notices placées à la fin des annales et des biographies et introduites par des formules du type «l'historien dit:» (shi chen yue 史臣曰). C'est le lieu où, en quelques phrases, l'historien livre son sentiment général sur la personne et sur sa conduite morale. 
C'est le cas par exemple lorsque Ban Gu 班固 fait une critique acerbe de Jia Yi 賈誼 [...] ou encore lorsque Wei Shou dit d'Erzhu Rong qu'il pourrait être comparé à [Yi] Yin ou Huo [Guang] 伊霍. Les uns sont contraires à la vérité, les autres se livrent à des rapprochements abusifs. Si l'on voulait critiquer toutes les erreurs de ce type, cinq chariots de livres n'y suffiraient pas. ${ }^{109}$

Une question se pose : pourquoi Wei Shou a-t-il autant défendu la mémoire d'Erzhu Rong ? A priori, ce dernier aurait pu être présenté comme un rebelle ou du moins traité de façon moins partisane, tel est le cas dans l'Histoire des dynasties du Nord et dans le Mémoire sur les monastères bouddhiques de Luoyang ${ }^{110}$. Pour Liu Zhiji, la réponse est simple : dans une note - c'est la seconde référence à la question d'Erzhu Rong - il rapporte, en passant, un propos du Livre des Qi du Nord selon lequel Wei Shou aurait été corrompu par l'un des fils de Rong afin qu'il compose une biographie flatteuse de son père ${ }^{111}$.

Cette histoire est peut-être vraie, mais il peut également s'agir d'une diffamation destinée soit à décrédibiliser le texte de Wei Shou, soit à expliquer rapidement et facilement le traitement d'Erzhu Rong. On retrouve une histoire semblable concernant Chen Shou, qui, dans la Monographie des Trois Royaumes, n'a pas accordé de biographie à des membres d'un clan pourtant influent, les Ding. Derrière l'histoire de corruption se cachait probablement une situation plus complexe, les Ding ayant été partisans de Cao Shuang, un adversaire des Sima ${ }^{112}$. Dans le cas d'Erzhu Rong, il pourrait s'agir d'une question de lien familial, comme l'a expliqué Zhao Yi ${ }^{113}$. Erzhu Rong souhaitait marier sa fille à Yuan Ziyou. Finalement, elle plut à Gao Huan, qui la prit pour concubine, de même qu'il prit pour concubine la fille d'Erzhu Zhao 爾朱兆 (†532), le neveu d'Erzhu Rong,

${ }^{109}$ STTS, A.9.77.

${ }^{110}$ Beishi, juan 48, p. 1776 ; Luoyang qielanji, loc. cit.

${ }^{111}$ STTS, A.21.148. Cf. Bei Qishu, juan 48, p. 667 et Beishi, juan 48, p. 1764.

${ }^{112}$ Cf. Damien Chaussende, Des Trois Royaumes aux Jin, p. 65-66.

${ }^{113}$ Chapitre 170 « La biographie d'Erzhu Rong » (爾朱榮傳) dans Nian'er shi zhaji jiaozheng, juan 13, p. 265. 


\section{Damien Chaussende}

qu'il défit à la bataille de Hanling ${ }^{114}$. Erzhu Rong n'ayant pas été tué par Gao Huan et étant le père de l'une de ses concubines préférée, Wei Shou put, et sans doute dut, lui composer une biographie positive, à l'inverse des autres membres du clan, qu'il présenta comme relativement avides de pouvoir, ce qui causa leur chute ${ }^{115}$.

Incontestablement, le Livre des Wei contraste avec les autres histoires du haut Moyen Âge : Wei Shou innove beaucoup tant dans la forme que dans le fond; ses intitulés surprenants, son dénigrement acerbe des pouvoirs du Sud, sa valorisation insistante du Nord en général et des Wei en particulier en sont les manifestations les plus évidentes. Il n'en demeure pas moins que Wei Shou s'inscrit dans la tradition historiographique officielle de son temps. Son œuvre, commandée par le pouvoir politique en place, avait une fonction politique bien précise : légitimer les Wei face aux États du Sud, et montrer que les Qi du Nord étaient les successeurs naturels et inéluctables des Wei occidentaux.

Nous prenons la pleine mesure de ce soubassement idéologique grâce, entre autres, aux commentaires de Liu Zhiji. L'ensemble de son traité, outre son intérêt propre, est un outil précieux pour discerner les dissemblances historiographiques entre les différentes sources textuelles du haut Moyen Âge. En un sens, et à sa façon, Liu Zhiji en donne quelquesunes des clés de lecture. En confrontant le critique à l'historien Wei Shou, on constate à quel point, en Chine, l'histoire est susceptible d'être réécrite en fonction des besoins politiques et des présupposés idéologiques des auteurs : 1'histoire est une recréation du passé destinée à servir le présent. La vulgate écrite par Wei Shou fut jugée inacceptable par Liu Zhiji, qui considérait le Sud seul comme légitime. Il tenait cette vérité pour acquise et allant de soi, ce qui explique en grande partie sa détestation de Wei Shou. Par ailleurs, il est pour le moins étonnant de constater que Liu Zhiji n'évoque pratiquement pas les deux histoires de Li Yanshou, qui ont pour originalité de placer sur le même plan les États du Sud et ceux du Nord : il ne leur consacre aucune notice dans le chapitre B.2, « Histoires officielles du passé et du présent », et ne les évoque guère qu'en passant, à la suite

\footnotetext{
${ }^{114}$ On trouve la biographie de cette dame dans Beishi, juan 14, p. 518.

${ }^{115}$ Voir le jugement récapitulatif de Wei Shou sur les membres du clan Erzhu (Erzhu Rong mis à part), Weishu, juan 75, p. 1677.
} 
des Mémoires historiques de Sima Qian, dans le chapitre A.1, « Les six écoles » (liujia 六家) ${ }^{116}$, où il n'en dit pas du bien. Critique virulente de Wei Shou, silence presque total sur Li Yanshou : Liu Zhiji - on l'a vu maintes fois en outre dans ses commentaires - a, tout comme Wei Shou, une conception bien marquée de l'histoire du haut Moyen Âge et surtout de la façon dont on doit la relater.

Est-ce à dire que l'historien moderne qui souhaite reconstituer l'enchaînement des événements politiques doive rejeter les sources textuelles, au motif que celles-ci ne seraient que pure propagande idéologique ? Outil de propagande, les histoires officielles le sont incontestablement. Mais elles ne sont évidemment pas non plus sans rapport avec la réalité des faits. L'histoire de Wei Shou, malgré tous ses défauts, et malgré le fait qu'elle légitimait les Qi du Nord, continua à être lue et utilisée sous les Tang, tandis que celle de Wei Dan, idéologiquement plus correcte (du point de vue des Tang, puisqu'elle légitimait les Zhou) finit par se perdre. Comme l'a noté Zhao Yi, c'est essentiellement en puisant chez Wei Shou, et non chez Wei Dan, que Li Yanshou écrivit son histoire ${ }^{117}$. C'est que le Livre des Wei, dans son récit événementiel, dans les faits qu'il décrit, dans les textes qu'il cite, est globalement crédible. Les sources historiques anciennes, et en particulier les histoires dynastiques, sont en effet fondées en grande partie sur des pièces officielles (chroniques de cour, édits impériaux, mémoires au trône...), au point que certaines parties, comme les annales, et dans une moindre mesure les biographies, s'apparentent à ce que les médiévistes occidentalistes appelleraient des regestes ${ }^{118}$, des répertoires chronologiques rassemblant des documents.

L'amour de la pièce officielle est l'un des traits saillants de l'historiographie chinoise, et une aubaine pour l'historien moderne. On pourrait, dans une attitude hypercritique, considérer que les textes officiels rappor-

${ }^{116}$ STTS, A.1.17-18.

${ }^{117} C f$. le chapitre 173 « Le "Livre des Wei" de l'Histoire des dynastie du Nord est essentiellement fondé sur l'ouvrage de Wei Shou » (北史魏書多以魏收書為本) dans Nian'er shi zhaji jiaozheng, juan 13, p. 269.

${ }^{118}$ Liu Zhiji donne d'ailleurs, dans le chapitre B.6, "Pointer l'encombrant » (Dianfan 點煩, STTS, B.6.410-411), des consignes sur la bonne façon de tronquer un édit afin d'en extraire ce qui mérite d'apparaître dans une histoire. 
tés sont forgés, que les faits relatés sont inventés, et que les auteurs de nos sources mentent. Certes, mais ils ne mentent pas tous sur les mêmes points. Aussi pouvons-nous, comme je l'ai fait au long de cet article, confronter les sources les unes aux autres. Lorsque plusieurs convergent, se complètent et s'éclairent, on peut penser tenir un fragment de la réalité factuelle. Or l'historien est bien doté pour le haut Moyen Âge ; sans compter l'apport de l'archéologie et de l'épigraphie, la documentation est très riche, et dépasse de loin celle des époques précédentes : pour une période de 350 ans environ (de 220 à 589), nous disposons de onze histoires officielles, composées le plus souvent dans des conditions politiques différentes, d'où des intentions idéologiques distinctes.

Pour mener à bien une recherche sur l'histoire du haut Moyen Âge, il est donc impératif d'étudier de manière approfondie le contexte de l'élaboration des histoires dynastiques, qui demeurent, pour cette période, les sources textuelles principales en matière d'histoire politique. Il faut avoir conscience que le corpus des vingt-cinq histoires, que nous voyons aujourd'hui au premier abord comme un ensemble harmonieux, s'est constitué au cours des siècles en réunissant des textes forts différents, dont les points communs n'étaient rien d'autre que la structure - la forme annales-biographies - et le fait d'avoir été agréé par le pouvoir impérial à une époque donnée ${ }^{119}$.

${ }^{119}$ Sur la constitution des quinze premiers titres de ce corpus, $c f$. K. H. J. Gardiner, "Standard Histories, Han to Sui", in Leslie Donald Daniel, Mackerras Colin and Wang Gungwu (ed.), Essays on the Sources for Chinese History, Canberra: National University Press, 1973, p. 42-52. 